TRANSACTIONS OF THE

AMERICAN MATHEMATICAL SOCIETY

Volume 357, Number 7, Pages 2535-2556

S 0002-9947(05)03212-5

Article electronically published on March 2, 2005

\title{
A RIGID SUBSPACE OF THE REAL LINE WHOSE SQUARE IS A HOMOGENEOUS SUBSPACE OF THE PLANE
}

\author{
L. BRIAN LAWRENCE
}

ABSTRACT. Working in ZFC, we give an example as indicated in the title.

\section{INTRODUCTION}

Recall that a topological space $X$ is homogeneous if for all $p, q \in X$, there exists an onto homeomorphism $H: X \rightarrow X$ such that $H(p)=q$; and at the opposite extreme, a space is rigid if the only bijective homeomorphism is the identity function. In [1, Jan van Mill constructed a rigid subspace of the Hilbert cube whose square is homeomorphic to the Hilbert cube, and therefore, homogeneous. He then asked whether there is a rigid subspace of the real line with a homogeneous square. Our answer is yes in ZFC.

Theorem. There is a ZFC example of a dense rigid subspace of the real line whose square is a dense homogeneous subspace of the plane.

\section{OUtLine OF THE PROOF}

2.1. Notation. In this subsection, we introduce just enough notation to give the strategy for the proof of the theorem.

\section{Standard notation:}

(1) Let $\mathcal{P}_{1}$ denote $\omega^{\omega}=\{f \quad \mid f: \omega \rightarrow \omega\}$ with the product topology of pointwise convergence ( $\omega$ denotes the set of all nonnegative integers); therefore, $\mathcal{P}_{1}$ is homeomorphic to the subspace of the real line consisting of all irrational numbers.

(2) For a function $f, \operatorname{dom}(f)$ and $\operatorname{ran}(f)$ denote the domain and range respectively.

(3) The cardinality operator is denoted by vertical bars.

(4) A single vertical bar denotes function restriction; and a small raised circle denotes composition of functions.

(5) The symbol $\mathbf{c}$ denotes the cardinality of the continuum of real numbers.

(6) The symbol for the empty set, $\phi$, also denotes the empty function as a root of a tree partially ordered by function extension.

(7) For a set $A, A^{2}$ denotes the square $A \times A$, the cartesian product of $A$ with itself, and $I d$ denotes the identity function on $A$.

(8) A slanted bar is used for the set difference operator $A \backslash B=\{x: x \in A \& x$ $\notin B\}$.

Received by the editors September 27, 2000.

2000 Mathematics Subject Classification. Primary 54B10; Secondary 54B05, 54C20.

Key words and phrases. Real line, plane, subspace, product, power, rigid, homogeneous.

(C)2005 American Mathematical Society 
Definition: Spaces and trees. We will let $X_{\mathbf{c}}$ denote our objective rigid subspace of $\mathcal{P}_{1}$ with homogeneous square.

Let $\mathcal{P}_{2}=\left\{f \mid f: \omega \rightarrow \omega^{2}\right\}$, with the product topology (so $\mathcal{P}_{2}$ is homeomorphic to $\mathcal{P}_{1}$ ); we will use $\mathcal{P}_{2}$ as a convenient representation for the square of $\mathcal{P}_{1}$.

Let $\mathcal{D} \subseteq \mathcal{P}_{1}$ be a fixed countable dense subset of $\mathcal{P}_{1} ; \mathcal{D}$ will serve as the starting point for constructing $X_{\mathbf{c}}$ (i.e., $\mathcal{D} \subseteq X_{\mathbf{c}}$ ).

Let $\Sigma=\{\sigma:[0, n] \rightarrow \omega \mid n \in \omega\} \cup\{\phi\}$ and let $\Lambda=\left\{\lambda:[0, n] \rightarrow \omega^{2} \mid n \in \omega\right\} \cup\{\phi\}$, each partially ordered by function extension (so each is a tree where $\phi$ is the root and each point has a countably infinite number of immediate successors). The height of a point in $\Sigma$ or $\Lambda$ is the cardinality of its domain. For each $n \in \omega$, the $n$th level of either tree is the subset consisting of all points with height $n$ (so each of our trees has a countably infinite number of levels). For each $\sigma \in \Sigma$ (respectively, $\lambda \in \Lambda$ ), let $\operatorname{ext}(\sigma)$ (respectively, $\operatorname{ext}(\lambda)$ ) denote the set of all extensions of $\sigma,\{\tau \in \Sigma: \tau \supseteq \sigma\}$ (respectively, extensions of $\lambda,\{\gamma \in \Lambda: \gamma \supseteq \lambda\}$ ), partially ordered by function extension; so each is a tree with roots $\sigma$ and $\lambda$ respectively.

Let $\mathcal{B}(\sigma)=\left\{f \in \mathcal{P}_{1}: \sigma \subseteq f\right\}$ and $\mathcal{B}(\lambda)=\left\{f \in \mathcal{P}_{2}: \lambda \subseteq f\right\}(\sigma \in \Sigma, \lambda \in \Lambda)$ (the values of $\mathcal{B}$ provide the basic open sets for our two topological spaces).

Definition: Project, adjoin, and combine. Relative to a cartesian product with two factors, $\pi_{1}$ and $\pi_{2}$ are the projection functions to the first and second coordinates respectively.

Suppose $\sigma \in \Sigma$ (respectively, $\lambda \in \Lambda$ ), and $m \in \omega$ (respectively, $t \in \omega^{2}$ ). Then $\sigma^{\wedge} m$ (respectively, $\lambda^{\wedge} t$ ) denotes $\sigma \cup\{\langle$ height $(\sigma), m\rangle\}$ (respectively, $\lambda \cup$ $\{\langle h e i g h t(\lambda), t\rangle\})$. If $\langle\sigma, \tau\rangle \in \Sigma^{2}$ with height $(\sigma)=h e i g h t(\tau)=n$, then $\ulcorner\sigma, \tau\urcorner$ denotes $\lambda:[0, n-1] \rightarrow \omega^{2}$ defined by $\lambda(m)=\langle\sigma(m), \tau(m)\rangle$. Similarly, if $\langle f, g\rangle \in \mathcal{P}_{1}^{2}$, then $\ulcorner f, g\urcorner$ denotes $h \in \mathcal{P}_{2}$ defined by $h(n)=\langle f(n), g(n)\rangle$. For $X \times Y \subseteq \mathcal{P}_{1}^{2}$,

$$
\ulcorner X \times Y\urcorner=\{\ulcorner f, g\urcorner:\langle f, g\rangle \in X \times Y\} .
$$

These are the adjoin and combine operations respectively.

Definition: Block functions and the tilde operator. Fix $\sigma \in \Sigma$. A function $\chi: \operatorname{ext}(\sigma) \rightarrow \Sigma$ is a block function iff height $(\chi(\sigma))=\operatorname{height}(\sigma) \& \forall \tau \supseteq$ $\sigma \exists c p(\chi, \tau): \omega \rightarrow \omega \forall n \in \omega\left[\chi\left(\tau^{\wedge} n\right)=\chi(\tau) \wedge c p(\chi, \tau)(n)\right]$. The functions $c p(\chi, \tau)(\tau \subseteq \sigma)$ are the components of $\chi$. A block function is completely determined by its value at the root (of its subtree domain) and its component functions. A block function defined on $\Sigma$ necessarily fixes the root $\phi$. Similarly, for a fixed $\lambda \in \Lambda$, a function $\psi: \operatorname{ext}(\lambda) \rightarrow \Lambda$ is a block function iff height $(\psi(\lambda))=h e i g h t(\lambda) \& \forall \gamma \supseteq$ $\lambda \exists c p(\psi, \gamma): \omega^{2} \rightarrow \omega^{2} \forall t \in \omega^{2}\left[\psi\left(\gamma^{\wedge} t\right)=\psi(\gamma)^{\wedge} c p(\psi, \gamma)(t)\right]$.

Note that block functions are simply those functions that are level and orderpreserving with respect to the tree structures; so a block function is a subtree order-isomorphism whenever each component function is a permutation.

For all $\lambda \in \Lambda$ (respectively, $\sigma \in \Sigma$ ), and for all block functions $\psi: \operatorname{ext}(\lambda) \rightarrow \Lambda$ (respectively, $\chi: \operatorname{ext}(\sigma) \rightarrow \Sigma$ ), define $\tilde{\psi}: \mathcal{B}(\lambda) \rightarrow \mathcal{P}_{2}$ by $\tilde{\psi}(f)=\bigcup_{n \in \omega} \psi(f \mid[0, n])$ (respectively, $\tilde{\chi}: \mathcal{B}(\sigma) \rightarrow \mathcal{P}_{1}$ by $\left.\tilde{\chi}(f)=\bigcup_{n \in \omega} \chi(f \mid[0, n])\right)$.

Definition: Homeomorphisms on our dense subset of the irrationals. Let $\mathcal{H}=\{H: H$ is a homeomorphism \& $\operatorname{dom}(H)=\mathcal{D} \& \operatorname{ran}(H)$ is a dense subset of $\left.\mathcal{P}_{1}\right\}$. Note that $|\mathcal{H}|=\mathbf{c}$. 
Definition: Bounded cartesian product. Let $E_{0}$ denote the set of all multiples of three in $\omega$. Suppose that $\forall n \in \omega$, factor $(n) \subseteq \omega$. Then the cartesian product $\Pi_{n \in \omega}$ factor $(n) \subseteq \mathcal{P}_{1}$ is bounded (or more accurately, has projections in $E_{0}$ with uniformly bounded cardinalities) if and only if $\exists m \in \omega \forall n \in \omega\left[\mid\right.$ factor $(n) \cap E_{0} \mid$ $\leq m]$.

2.2. Strategy for the proof. The strategy is intended to serve as both an introduction to the proof at the start, and as a consolidation of the proof at the end.

We will construct our objective subspace of the real line as $X_{\mathbf{c}}=C l_{\mathcal{G}}\left(\mathcal{D} \cup\left\{p_{\alpha}\right.\right.$ : $\alpha \in \mathbf{c}\}$ ), where $C l_{\mathcal{G}}$ is the $\mathcal{G}$ closure operator ( $\mathcal{G}$ is a group of order-isomorphisms on $\Lambda$; more details are given below under Objective of Lemma 2), which takes subsets of the line as arguments and returns a subset of the line that both extends the original, and, has a homogeneous square in the plane. The function $\left\langle p_{\alpha}\right.$ : $\alpha \in \mathbf{c}\rangle$ is one-to-one and defined recursively. Since $\mathcal{D}$ is a dense subset of $X_{\mathbf{c}}$, any potential homeomorphism of $X_{\mathbf{c}}$ onto $X_{\mathbf{c}}$ is a continuous extension of some member of $\mathcal{H}$. We define below, for each $H \in \mathcal{H}$, a continuous extension $H^{e}$ of $H$ such that $\operatorname{dom}\left(H^{e}\right)$ is an intersection of countably many dense open subsets of $\mathcal{P}_{1}$, and therefore, has cardinality $\mathbf{c}$ in every open interval. Our first step is to well-order $\mathcal{H} \backslash\{$ Identity Function on $\mathcal{D}\}$ as $\left\{H_{\alpha}: \alpha \in \mathbf{c}\right\}$. At each stage $\alpha \in \mathbf{c}$, we define $X_{\alpha}=C l_{\mathcal{G}}\left(\mathcal{D} \cup\left\{p_{\beta}: \beta<\alpha\right\}\right)$, and take as our Recursion Hypothesis that $\left\{p_{\beta}: \beta<\alpha\right\}$ has been constructed so that $X_{\alpha}$ is disjoint from $\left\{q_{\beta}: \beta<\alpha\right\}$. We then introduce a new point $p_{\alpha} \in \operatorname{dom}\left(H_{\alpha}^{e}\right)$ and define $q_{\alpha}=H_{\alpha}^{e}\left(p_{\alpha}\right)$. We use a construction that guarantees (more details are given below) that $p_{\alpha}$ and $q_{\alpha}$ are distinct points that are outside the union of $X_{\alpha}$ and $\left\{q_{\beta}: \beta<\alpha\right\}$, and such that $X_{\alpha+1}$ and $\left\{q_{\beta}: \beta \leq \alpha+1\right\}$ are disjoint. Also, in the end, we have that $X_{\mathbf{c}}=\bigcup_{\alpha<\mathbf{c}} X_{\alpha}$. From these claims we get that $X_{\mathbf{c}}$ and $\left\{q_{\alpha}: \alpha<\mathbf{c}\right\}$ are disjoint; and therefore, for every $\alpha \in \mathbf{c},\left\langle p_{\alpha}, q_{\alpha}\right\rangle$ is a witness for the failure of $H_{\alpha}$ to extend to a homeomorphism of $X_{\mathbf{c}}$ onto $X_{\mathbf{c}}$. Thus, $X_{\mathbf{c}}$ is rigid; and since $X_{\mathbf{c}}$ is the result of applying the $\mathcal{G}$ closure operator, $X_{\mathbf{c}} \times X_{\mathbf{c}}$ is homogeneous.

Objective of Lemma 1. In order to carry out the program outlined above, our first step is to give a procedure that takes an ordinal $\alpha \in \mathbf{c}$ and a basic open subset $U$ of $\mathcal{P}_{1}$ as arguments and returns a set value $T \subseteq U \cap \operatorname{dom}\left(H_{\alpha}^{e}\right)$ with $|T|=\mathbf{c}$, and such that each of $T$ and $H_{\alpha}^{e}[T]$ has finite intersection with every bounded cartesian product.

Objective of Lemma 2. The next step is to construct a countably infinite collection of permutations on $\omega^{2}$, denoted by $\Delta$, such that $\Delta$ forms a group under composition of functions. We then define an operator $\theta$ (at the level of $\omega$ ) such that for each $i \in\{1,2\}$, for each $\eta \in \Delta$, and for all $f, g: \omega \rightarrow \omega, \theta(i, \eta, f, g)=h$ where $h: \omega \rightarrow \omega$ defined by $h(n)=\pi_{i}(\eta(f(n), g(n)))$. A standard composition over $\omega$ is defined recursively by taking the identity on $\omega$ and all constant functions on $\omega$ with an integer value as the ground set. Then an arbitrary standard composition is obtained from the ground set by repeated applications of $\theta$. At the next level, we construct a countably infinite collection of order-isomorphisms on $\Lambda$, denoted by $\mathcal{G}$, such that $\mathcal{G}$ also forms a group under composition of functions. Each component function of each order-isomorphism in $\mathcal{G}$ is a composition involving members of $\Delta$ and two-point interchanges on $\omega^{2}$. For each $\psi \in \mathcal{G}, \tilde{\psi}$ is 
a homeomorphism on the plane. Let $\tilde{\mathcal{G}}=\{\tilde{\psi}: \psi \in \mathcal{G}\}$. Then $\langle\tilde{\mathcal{G}}, 0\rangle$ is a group of homeomorphisms on $\mathcal{P}_{2}$ with $|\tilde{\mathcal{G}}|=\omega$. We now have groups at the level of $\omega^{2}, \Lambda$, and $\mathcal{P}_{2}$. The $\mathcal{G}$ closure of a subset $Z$ of $\mathcal{P}_{1}$ with $Z \supseteq \mathcal{D}$ is defined to be $\bigcap\left\{Y \subseteq \mathcal{P}_{1}: Z \subseteq Y \& \forall \psi \in \mathcal{G}(\tilde{\psi}[[Y \times Y]] \subseteq[Y \times Y])\right\}$. Note that $\left|C l_{\mathcal{G}}(Z)\right|=|Z|$ (since $\mathcal{G}$ is countable). By Lemma 2 , for every subset $Z$ of $\mathcal{P}_{1}$ with $Z \supseteq \mathcal{D}$, the $\mathcal{G}$ closure of $Z$ has for its square, a dense homogeneous subspace of the plane. A homeomorphism $F$ that serves as one of the witnesses for homogeneity is defined by choosing a sequence of homeomorphisms in $\mathcal{G},\left\langle\psi_{n}: n \in \omega\right\rangle$, and, for each $f \in \operatorname{dom}(F)$, setting $F(f)$ equal to the pointwise limit of $\left\langle\tilde{\psi}_{n}(f): n \in \omega\right\rangle$, where, with the exception of the two points being interchanged, each value is obtained as the limit of an eventually constant point sequence (this last part guarantees that $F$ maps the square of the $\mathcal{G}$ closure (of a set) back into the square).

2.3. Objective of Lemma 3. The third step is to introduce the notion of a standard composition on a basic open subset of $\mathcal{P}_{1}$ for the purpose of characterizing the $\mathcal{G}$ closure operator. Define $\theta$ (at the level of $\mathcal{P}_{1}$ ) as follows: for each $i \in\{1,2\}$, and for each $\psi \in \mathcal{G}$, and for each $\sigma \in \Sigma$, and for all $F, G: \mathcal{B}(\sigma) \rightarrow \mathcal{P}_{1}$, we define $\theta(i, \psi, F, G)=H$ where $\operatorname{dom}(H)=\mathcal{B}(\sigma) \& H(h)=\pi_{i} \circ(\tilde{\psi}([F(h), G(h)]))$; note that $\psi([F(h), G(h)])$ is a function from $\omega$ into $\omega^{2}$, and $\pi_{i}$ is a function from $\omega^{2}$ into $\omega$. Suppose $\mathcal{D} \subseteq Z \subseteq \mathcal{P}_{1}$. Then the set of all standard compositions determined by $Z$, denoted $s c(Z)$, is defined to be the union over all $\sigma \in \Sigma$ of the following recursively defined collection: the ground set contains the identity function on $\mathcal{B}(\sigma)$ and every constant function on $\mathcal{B}(\sigma)$ where the value is a member of $Z$. Then an arbitrary standard composition on $\mathcal{B}(\sigma)$ determined by $Z$ is constructed from the ground set by repeated applications of $\theta$ (this definition requires a minor modification, as explained in Section 7, that is irrelevant for our current purpose). Each standard composition on $\mathcal{B}(\sigma)$ is a continuous function, and is induced by a block function $\chi: \operatorname{ext}(\sigma) \rightarrow \Sigma$, where each component is a standard composition on $\omega$. The set of all special operators determined by $Z$, denoted $\mathcal{O}(Z)$, consists of all standard compositions determined by $Z$ for which each component of the underlying block function is neither the identity on $\omega^{2}$ nor a constant function on $\omega^{2}$. Note that $|\mathcal{O}(Z)|=|Z|$. By Lemma 3, for all $Z$ such that $\mathcal{D} \subseteq Z \subseteq \mathcal{P}_{1}$, and for every $f \in \mathcal{P}_{1}, C l_{\mathcal{G}}(Z \cup\{f\}) \subseteq C l_{\mathcal{G}}(Z) \cup\{f\} \cup\left\{F(f): F \in \mathcal{O}\left(C l_{\mathcal{G}}(Z)\right) \& f \in \operatorname{dom}(F)\right\}$. In our construction above (first paragraph of this subsection), $Z$ and $f$ vary over $X_{\alpha}$ and $p_{\alpha}$ respectively as $\alpha$ varies over c. (Also note that Lemma 3 verifies that for every $\alpha \in \mathbf{c}, X_{\alpha}$ has cardinality less than $\mathbf{c}$.)

Objective of Lemma 4. The following result is the last step. Suppose $\mathcal{D} \subseteq Z \subseteq$ $\mathcal{P}_{1}$ and $F \in \mathcal{O}(Z)$. Suppose further that $Y$ is either the range of $F$ or a point-inverse set of $F$. Then $Y$ is a subset of a bounded cartesian product.

By Lemma 2, the square $X_{\mathbf{c}} \times X_{\mathbf{c}}$ is homogeneous. We now use Lemmas 1, 3, and 4 to justify that our Recursion Hypothesis is preserved in the argument above for constructing $X_{\mathbf{c}}$. Suppose $\alpha \in \mathbf{c}$. Let $U$ be an open interval such that $U \cap \mathcal{D}$ is disjoint from its image under $H_{\alpha}$. With $U$ and $\alpha$ as arguments, choose $T$ according to Lemma 1. Let $\mathcal{C}=\left\{\operatorname{ran}(F): F \in \mathcal{O}\left(X_{\alpha}\right)\right\} \cup\left\{F^{-1}\left(q_{\beta}\right): \beta<\alpha \& F \in \mathcal{O}\left(X_{\alpha}\right)\right\}$. Then the cardinality of $\mathcal{C}$ is less than $\mathbf{c}$ and, by Lemma 4 , each member of $\mathcal{C}$ is a subset of a bounded cartesian product. We can therefore choose $p_{\alpha} \in T$ so that each of $p_{\alpha}$ and $q_{\alpha}=H_{\alpha}^{e}\left(p_{\alpha}\right)$ resides in the complement of $\left((\cup \mathcal{C}) \cup X_{\alpha} \cup\left\{q_{\beta}: \beta<\alpha\right\}\right)$. By Lemma 3, the Recursion Hypothesis is preserved. 


\section{HomeOMORPHisms ON SUBSPACES OF THE LINE}

3.1. Continuous extensions. Suppose $H \in \mathcal{H}$ or $H^{-1} \in \mathcal{H}$, and $\sigma \in \Sigma$. Then define $b(H, \sigma)$ to be a subcollection of $\Sigma$ satisfying each of the following conditions:

(1) $\forall \tau \in b(H, \sigma)$, height $(\tau)>\operatorname{height}(\sigma)$;

(2) $\forall \tau_{1}, \tau_{2} \in b(H, \sigma)$, if $\tau_{1} \neq \tau_{2}$, then $\tau_{1}$ and $\tau_{2}$ are incomparable (i.e., neither function extends the other);

(3) $H[\mathcal{B}(\sigma) \cap \operatorname{dom}(H)]=\bigcup\{\mathcal{B}(\tau) \cap \operatorname{ran}(H): \tau \in b(H, \sigma)\}$.

Suppose $H \in \mathcal{H}$. Define the corresponding tree for $H$, denoted $\operatorname{tr}(H)$, by the following rules:

(1) the point-set of the tree is a subset of $\Sigma$;

(2) the set of all roots (level zero) is $\mathcal{L}_{0}=\{\sigma \in \Sigma: \operatorname{dom}(\sigma)=\{0\}\}$;

(3) if $\mathcal{L}_{n}$ denotes level $n$ of $\operatorname{tr}(H)$, then, $\forall \sigma \in \operatorname{tr}(H)$, the set of all immediate successors of $\sigma$ in the tree partial order is recursively defined to be $b(H, \sigma)$ if $\sigma \in \mathcal{L}_{n}$ where $n$ is even, and is $b\left(H^{-1}, \sigma\right)$ if $\sigma \in \mathcal{L}_{n}$ where $n$ is odd.

Let $X=\bigcap\left\{\bigcup\left\{\mathcal{B}(\sigma): \sigma \in \mathcal{L}_{2 n}\right\}: n \in \omega\right\}$ and let $Y=\bigcap\left\{\bigcup\left\{\mathcal{B}(\sigma): \sigma \in \mathcal{L}_{2 n+1}\right\}\right.$ : $n \in \omega\}$. Then there is a continuous extension of $H$, denoted $H^{e}$, such that $H^{e}$ is a homeomorphism of $X$ onto $Y$.

Note that there is a bijective correspondence between the set of all ordered pairs on the graph of $H^{e}$ and the set of all maximal branches of $\operatorname{tr}(H)$. Also note that for every open interval $U$ on the real line, $|X \cap U|=|Y \cap U|=\mathbf{c}$, since each of $X$ and $Y$ is the intersection of a countable collection of dense open subsets of $\mathcal{P}_{1}$.

3.2. Large sets of irrationals having finite intersection with every bounded cartesian product. We begin with a result that is of interest in its own right. The proof is due to Ronnie Levy. An extension of the Levy construction yields a proof of Lemma 1. The proof of Lemma 1 is self-contained; however, the reader is well advised to first read Levy's proof in preparation for the extension.

Preliminary Theorem for Lemma 1. A compact subset $C \subseteq \mathcal{P}_{1}$ is defined to be bounded iff $\exists n \in \omega \forall m \in \omega[|\{f(m): f \in C\}| \leq n]$ (i.e., there is a natural number $n$ such that for each projection function, the restriction of the projection function to $C$ has a range for which the cardinality is bounded by $n$ ). Then $\mathcal{P}_{1}$ is not the union of fewer than $\mathbf{c}$ bounded compact sets.

Claim. $\exists T \subseteq \mathcal{P}_{1}$ such that

(1) $|T|=\mathbf{c}$, and

(2) $\forall$ finite $S \subseteq T \exists m_{0} \in \omega\left[\left|\left\{f\left(m_{0}\right): f \in S\right\}\right|=|S|\right]$.

Proof of the Claim. Let $I$ be an independent family of cardinality c with ground set $\omega$ (i.e., $I$ is a cardinality $\mathbf{c}$ collection of infinite subsets of $\omega$ such that $\forall \mathcal{A}_{1}, \mathcal{A}_{2} \subseteq I$ with $\left.\mathcal{A}_{1} \neq \phi\left[\left|\mathcal{A}_{1}\right|<\omega \&\left|\mathcal{A}_{2}\right|<\omega \& \mathcal{A}_{1} \cap \mathcal{A}_{2}=\phi \Rightarrow\left|\cap \mathcal{A}_{1} \backslash \bigcup \mathcal{A}_{2}\right|=\omega\right]\right)$. Let $\rho$ be a bijection of $\mathbf{c} \times \omega$ onto $I$. Let $F: \mathbf{c} \rightarrow \mathcal{P}_{1}$ be defined by $F_{\alpha}(m)=n$ if $m \in$ $\rho(\alpha, n) \backslash \bigcup\{\rho(\alpha, k): k<n\}$, and $F_{\alpha}(m)=0$ if $m \in \omega \backslash \bigcup\{\rho(\alpha, k): k \in \omega\}$. Let $T=\operatorname{ran}(F)$. Let $l \in \omega$, and let $\beta:[0, l] \rightarrow \mathbf{c}$ be one-to-one. Let $m_{0} \in \bigcap\left\{\rho\left(\beta_{n}, n\right)\right.$ : $n \leq l\} \backslash \bigcup\left\{\rho\left(\beta_{n}, k\right): n \leq l \& k<n\right\}$. Then for every $n \leq l, F_{\beta_{n}}\left(m_{0}\right)=n$.

Proof of the Theorem. Note that the theorem follows immediately from the claim, since every bounded compact set has finite intersection with $T$. 
Lemma 1. Suppose $H \in \mathcal{H}, \nu \in \Sigma$ with $\nu \neq \phi$, and $j: \omega \rightarrow \omega$ is strictly increasing. Then $\exists T \subseteq \operatorname{dom}\left(H^{e}\right) \cap \mathcal{B}(\nu)$ such that

(1) $|T|=\mathbf{c}$, and,

(2) $\forall$ finite $S \subseteq T \exists m_{1}, m_{2} \in \omega$ such that

(2.1) $\left\{f\left(m_{1}\right): f \in S\right\} \cup\left\{f\left(m_{2}\right): f \in H^{e}[S]\right\} \subseteq \operatorname{ran}(j)$;

(2.2) $\left|\left\{f\left(m_{1}\right): f \in S\right\}\right|=\left|\left\{f\left(m_{2}\right): f \in H^{e}[S]\right\}\right|=|S|$.

Proof of Lemma 1. For every positive $n \in \omega$, let $\Gamma_{n}=\{\sigma \in \Sigma \mid \sigma:[0, n-1] \rightarrow$ $[0, n-1]\}$. Recursively construct a strictly increasing sequence $i: \omega \rightarrow \omega$ as follows: Let $\mu$ be an extension of $\nu$ such that $\mu \in \Gamma_{i(0)}$ where $i(0)=$ height $(\mu)$ (for instance, choose $\mu$ such that height $(\mu)=\max (\operatorname{ran}(\nu))+1$, and for each $n$ with height $(\nu) \leq n \leq \max (\operatorname{ran}(\nu)), \mu(n)=0)$. We now use the fact that each value of $\Gamma$ is finite to construct $i(n)$ for $n>0$. Choose $i(1)>i(0)$ such that $\forall \sigma \in \Gamma_{i(0)+1} \exists \tau \in \Gamma_{i(1)}[\tau$ extends a function in $b(H, \sigma)]$. Choose $i(2)>i(1)$ such that $\forall \sigma \in \Gamma_{i(1)+1} \exists \tau \in \Gamma_{i(2)}\left[\tau\right.$ extends a function in $\left.b\left(H^{-1}, \sigma\right)\right]$. Continue through $\omega$ stages, using $H$ for the definition of $i(n)$ if $n$ is odd, and using $H^{-1}$ if $n$ is even.

Let each of $I$ and $J$ be an independent family of cardinality $\mathbf{c}$ where the ground set for $I$ is $\{i(n): n$ is even $\}$ and the ground set for $J$ is $\{i(n): n$ is odd $\}$. Let each of $\rho_{1}: \mathbf{c} \times \omega \rightarrow I$ and $\rho_{2}: \mathbf{c} \times \omega \rightarrow J$ be a bijection. For each $\alpha \in \mathbf{c}$, let $F_{\alpha}$ be the partial (with respect to $\omega$ ) function defined by $F_{\alpha}(m)=j(n)$ if $m \geq j(n)$ and $m \in \rho_{1}(\alpha, n) \backslash \bigcup\left\{\rho_{1}(\alpha, k): k<n\right\}$, and $m \notin \operatorname{dom}\left(F_{\alpha}\right)$ otherwise. Note that $\operatorname{dom}\left(F_{\alpha}\right) \subseteq\{i(n): n$ is even $\}$, and for all $m \in \operatorname{dom}\left(F_{\alpha}\right), F_{\alpha}(m) \leq m$. Define $G_{\alpha}$ by replacing $\rho_{1}$ by $\rho_{2}$. Note that $\operatorname{dom}\left(G_{\alpha}\right) \subseteq\{i(n): n$ is odd $\}$, and for all $m \in \operatorname{dom}\left(G_{\alpha}\right), G_{\alpha}(m) \leq m$. Let $\Psi: \operatorname{ran}(F) \rightarrow \operatorname{ran}(G)$ be a bijection.

Claim. $\forall\langle f, g\rangle$ on the graph of $\Psi$ there exist total functions $f^{\prime}, g^{\prime} \in \mathcal{P}_{1}\left[f^{\prime} \supseteq\right.$ $\left.f \& g^{\prime} \supseteq g \& g^{\prime}=H^{e}\left(f^{\prime}\right)\right]$.

Proof of the Claim. We recursively construct finite restrictions of $f^{\prime}$ and $g^{\prime}$. Let $\sigma_{0} \in \Gamma_{i(0)+1}$ such that $\sigma_{0} \mid[0, i(0))=\mu$, and $\sigma_{o}(i(0))=f(i(0))$ if $f(i(0))$ is defined. Let $\tau_{0} \in \Gamma_{i(1)+1}$ such that $\tau_{0}$ extends a function in $b\left(H, \sigma_{0}\right)$ and $\tau_{0}(i(1))=g(i(1))$ if $g(i(1))$ is defined. Let $\sigma_{1} \in \Gamma_{i(2)+1}$ such that $\sigma_{1}$ extends a function in $b\left(H^{-1}, \tau_{0}\right)$ and $\sigma_{1}(i(2))=f(i(2))$ if $f(i(2))$ is defined. Continue through $\omega$ stages, using $f$ and $H^{-1}$ in the definition of $\sigma$, and using $g$ and $H$ in the definition of $\tau$. Let $f^{\prime}=\bigcup_{n \in \omega} \sigma_{n}$, and let $g^{\prime}=\bigcup_{n \in \omega} \tau_{n}$.

We now finish the proof of Lemma 1. Let $T=\left\{f^{\prime}: f \in \operatorname{ran}(F)\right\}$. For the verification of the intended properties, let $l \in \omega$, and let $\beta:[0, l] \rightarrow \mathbf{c}$ be one-toone. Let $m_{1} \in \omega$ such that $m_{1} \geq j(l)$ and $m_{1} \in \bigcap\left\{\rho_{1}\left(\beta_{n}, n\right): n \leq l\right\} \backslash \bigcup\left\{\rho_{1}\left(\beta_{n}, k\right)\right.$ : $n \leq l \& k<n\}$. Then $\forall n \leq l, F_{\beta_{n}}\left(m_{1}\right)=j(n)$. Choose $m_{2} \in \omega$ by the analogous formula where $\gamma \circ \beta$ replaces $\beta$ and $\rho_{2}$ replaces $\rho_{1}$, and with $\gamma: \mathbf{c} \rightarrow \mathbf{c}$ the bijection induced by $\Psi$.

\section{Homogeneous subspaces of the Plane}

4.1. Underlying permutation group on $\boldsymbol{\omega}^{2}$. Let $E_{0}=\{3 n: n \in \omega\} ; E_{1}=$ $\{3 n+1: n \in \omega\} ; E_{2}=\{3 n+2: n \in \omega\}$. Let $R=\left\{\langle n, n+1\rangle: n \in E_{1}\right\}$ ( $R$ is the raised diagonal set). Define a strict partial order $\prec$ on $\omega^{2}$ by the following rule: $s \prec t$ iff $\max \left\{\pi_{1}(s), \pi_{2}(s)\right\}<\min \left\{\pi_{1}(t), \pi_{2}(t)\right\}$. Note that $R \cup\left\{\langle n, n\rangle: n \in E_{0}\right\}$ is well-ordered by $\prec$. 
By recursion, we first construct a function $\delta: \omega \times \Lambda \times \omega^{2} \rightarrow \omega^{2}$ according to the following rules.

Well-order $E_{1} \times \Lambda \times \omega^{2}$ in type $\omega$. Suppose $\langle i, \lambda, t\rangle \in \omega \times \Lambda \times \omega^{2}$.

(1) If $i \in E_{0}$, then $\delta(i, \lambda, t)=t$.

(2) If $i=3 n+2$ for some $n \in \omega$, then $\delta(i, \lambda, t)=\delta(3 n+1, \lambda, t)$.

(3) Suppose $i \in E_{1}$, and $\delta(j, \rho, s)$ has been defined for every predecessor $\langle j, \rho, s\rangle$ of $\langle i, \lambda, t\rangle$ in the well-ordering of $E_{1} \times \Lambda \times \omega^{2}$. If there exists a predecessor $\langle j, \rho, s\rangle$ of $\langle i, \lambda, t\rangle$ such that $j=i, \rho=\lambda$, and $\delta(j, \rho, s)=t$, then define $\delta(i, \lambda, t)=s$. Otherwise, define $\delta(i, \lambda, t)=r$ where $r$ is the least (with respect to $\prec$ ) point in $R$ such that $t \prec r$, and, $s \prec r$ for each $s$ in the range of $\delta$ restricted to the predecessors of $\langle i, \lambda, t\rangle$ in the well-ordering of $E_{1} \times \Lambda \times \omega^{2}$. For each $\mu=\langle i, \lambda\rangle \in \omega \times \Lambda$, define $\delta_{\mu}: \omega^{2} \rightarrow \omega^{2}$ by $\delta_{\mu}(t)=\delta(i, \lambda, t)$. Then $\delta_{\mu}$ is a permutation on $\omega^{2}$ with $\delta_{\mu}=\delta_{\mu}^{-1}$ (and with $\delta_{\mu}$ the identity on $\omega^{2}$ whenever $\mu \in E_{0} \times \Lambda$ ).

Partition $E_{1} \times \Lambda \times \omega^{2}$ into two-point cells of the form $\{\langle i, \lambda, s\rangle,\langle i, \lambda, t\rangle\}$ such that $t=\delta(i, \lambda, s)$ and $s=\delta(i, \lambda, t)$ (of course, either equation implies the other). We now define the set of arguments for delta's forward action. Let $d f a=\{\langle i, \lambda, t\rangle \in$ $\left.E_{1} \times \Lambda \times \omega^{2}: t \prec \delta(i, \lambda, t)\right\}$. Then $d f a$ contains precisely one point of each (twopoint) cell in the partition. Define the forward action of $\delta$ to be the restriction $\delta \mid d f a$. Note that $\delta \mid d f a$ is a one-to-one function from $d f a$ into $R$.

Let $\Delta=\left\{\delta_{\mu_{0}} \circ \cdots \circ \delta_{\mu_{n}}: n \in \omega \& \mu:[0, n] \rightarrow \omega \times \Lambda\right\}$. Then $\langle\Delta, \circ\rangle$ is a group of permutations on $\omega^{2}$ with $|\Delta|=\omega$. Let $I d$ denote the identity permutation on $\omega^{2}$. For every $t \in \omega^{2}$, let $\operatorname{orbit}(t)=\{\eta(t): \eta \in \Delta\}$ (the $\Delta$ orbit of $t$ ). Note that $\left\{\right.$ orbit $\left.(t): t \in \omega^{2}\right\}$ is a partition of $\omega^{2}$. Fix $t \in \omega^{2}$. Let $s \in \operatorname{orbit}(t)$ be minimal with respect to $\prec$. We can now visualize $\operatorname{orbit}(t)$ as a tree, where the number of levels is infinite, the number of immediate successors of each point is infinite, the root of the tree is $s$, and for every $n \in \omega$, level $n+1$ is $\left\{\left(\delta_{\mu_{0}} \circ \cdots \circ \delta_{\mu_{n}}\right)(s) \mid \mu\right.$ : $\left.[0, n] \rightarrow E_{1} \times \Lambda \& \forall m<n\left[\delta_{\mu_{m}} \neq \delta_{\mu_{m+1}}\right]\right\}$. Since traveling on a branch of the tree away from $s$ captures the forward action of $\delta, s$ is the unique $\prec$ minimal element of $\operatorname{orbit}(t)$. Define the root function, denoted $r t$, by $r t(t)=s$ (i.e., $\forall t \in \omega^{2}, r t(t)$ is the least $\prec$ element of $\operatorname{orbit}(t)$; in particular, $r t$ is constant on each orbit). A point of $\omega^{2}$ is defined to be a root iff it is a fixed point of $r t$. Note that the range of $r t$ and the fixed point set of $r t$ coincide, each orbit contains exactly one root, and each point of $\omega^{2} \backslash R$ is a root.

For every $\lambda \in \Lambda$, define the subgroup of $\Delta$ determined by $\lambda$ to be

$$
\operatorname{subgroup}(\Delta, \lambda)=\left\{\delta_{\mu_{0}} \circ \cdots \circ \delta_{\mu_{n}}: n \in \omega \& \mu:[0, n] \rightarrow \omega \times\{\lambda\}\right\}
$$

(with composition of functions the group operation).

Fact 1. The group $\langle\Delta, 0\rangle$ possesses each of the following properties:

(1) each element of $\Delta \backslash\{I d\}$ has a unique (standard) representation as $\delta_{\mu_{0}} \circ \cdots \circ$ $\delta_{\mu_{n}}$ where $n \in \omega$ and $\mu:[0, n] \rightarrow E_{1} \times \Lambda$ such that $\forall m \in[0, n+1), \mu_{m} \neq \mu_{m+1}$ (and therefore, $\delta_{\mu_{m}} \neq \delta_{\mu_{m+1}}$ );

(2) $\omega^{2}$ is the disjoint union of the range of $\delta \mid d f a$ and the range of $r t$;

(3) $\forall t \in \omega^{2}[\operatorname{orbit}(t) \subseteq R \cup\{r t(t)\}]$; and for each root $t: \forall \eta, \xi \in \Delta \backslash\{I d\}$ with $\eta \neq \xi[\eta(t)$ and $\xi(t)$ are distinct points in $R]$;

(4) $\forall \lambda_{1}, \lambda_{2} \in \Lambda$ with $\lambda_{1} \neq \lambda_{2}, \operatorname{subgroup}\left(\Delta, \lambda_{1}\right) \cap \operatorname{subgroup}\left(\Delta, \lambda_{2}\right)=\{I d\}$.

Proof of Fact 1. The first three clauses follow from the fact that $\delta \mid d f a$ is a oneto-one function from $d f a$ into $R$, and from the tree structure that we imposed on each of the $\Delta$ orbits. The fourth clause follows from the first. 
Remark. For each fixed $i \in \omega, \delta$ induces an order-isomorphism $\psi: \Lambda \rightarrow \Lambda$ by defining, for each $\lambda \in \Lambda, c p(\psi, \lambda)(t)=\delta(i, \lambda, t)$. The next step is to modify these order-isomorphisms for the purpose of constructing homogeneous subspaces of $\mathcal{P}_{2}$.

4.2. Underlying permutation group on $\boldsymbol{\Lambda}$. We now use $\delta$ to construct a group of permutations on $\Lambda$.

For all $s, t \in \omega^{2}$, define $s$ lex $t$ to mean that $s$ is less than $t$ in the lexicographical (linear) ordering of $\omega^{2}$ (i.e., $\pi_{1}(s)<\pi_{1}(t)$ or $\left[\pi_{1}(s)=\pi_{1}(t) \& \pi_{2}(s)<\pi_{2}(t)\right]$ ).

Let $\varepsilon: \omega \rightarrow \Lambda \times \omega^{2} \times \omega^{2}$ be a bijection such that

(i) $\varepsilon\left[E_{0}\right]=\left\{\langle\lambda, s, t\rangle \in \Lambda \times \omega^{2} \times \omega^{2}: s=t\right\}$;

(ii) $\varepsilon\left[E_{1}\right]=\left\{\langle\lambda, s, t\rangle \in \Lambda \times \omega^{2} \times \omega^{2}: s\right.$ lex $\left.t\right\}$;

(iii) $\forall n \in \omega[\varepsilon(3 n+1)=\langle\lambda, s, t\rangle \Rightarrow \varepsilon(3 n+2)=\langle\lambda, t, s\rangle]$.

For each $i \in \omega$, let $\varphi_{i}: \Lambda \rightarrow \Lambda$ be the block function defined as follows. Let $\left\langle\lambda_{0}, s_{0}, t_{0}\right\rangle=\varepsilon(i)$. Suppose $\lambda \in \Lambda$ and $t \in \omega^{2}$. Then

(i) $c p\left(\varphi_{i}, \lambda_{0}\right)\left(s_{0}\right)=t_{0}$ and $c p\left(\varphi_{i}, \lambda_{0}\right)\left(t_{0}\right)=s_{0}$;

(ii) $c p\left(\varphi_{i}, \lambda_{0}\right)(t)=t$ if $t \notin\left\{s_{0}, t_{0}\right\}$;

(iii) $c p\left(\varphi_{i}, \lambda\right)(t)=\delta(i, \lambda, t)$ if $\lambda$ is a proper extension of $\lambda_{0}$;

(iv) $c p\left(\varphi_{i}, \lambda\right)(t)=t$ if $\lambda \notin \operatorname{ext}\left(\lambda_{0}\right)$.

Note that if $\varepsilon(i)=\langle\lambda, s, t\rangle$ and $s=t$, then $\varphi_{i}$ is the identity function on $\Lambda$; and if $\varepsilon(i)=\langle\lambda, s, t\rangle$ and $s$ lex $t$, then $\varphi_{i}=\varphi_{i+1}$ (i.e., the permutation on $\Lambda$ that $\varphi$ associates with $\langle\lambda, s, t\rangle$ is the same as the one that $\varphi$ associates with $\langle\lambda, t, s\rangle)$.

Each $\varphi_{i}$ is a permutation (in fact, an order-isomorphism) on $\Lambda$, since each of its components is a permutation on $\omega^{2}$, and for each $i \in \omega, \varphi_{i}=\varphi_{i}^{-1}$ since each component satisfies this equation.

Fact 2. Let $\mathcal{G}=\left\{\varphi_{i_{0}} \circ \cdots \circ \varphi_{i_{n}}: n \in \omega \& i:[0, n] \rightarrow \omega\right\}$. Then $\langle\mathcal{G}, \circ\rangle$ is a group of order-isomorphisms on $\Lambda$, with $|\mathcal{G}|=\omega$, such that each of the following conditions is satisfied:

(1) Suppose $\psi \in \mathcal{G}, n \in \omega$ and $i:[0, n] \rightarrow \omega$ such that $\psi=\varphi_{i_{0}} \circ \cdots \circ \varphi_{i_{n}}$. Let $l=\max \left\{\right.$ height $(\lambda)+1: \exists m \leq n$ such that $\lambda$ is the first component of $\left.\varepsilon\left(i_{m}\right)\right\}$. Then $\forall \lambda \in \Lambda$ with height $(\lambda)=l: \forall \gamma \supseteq \lambda[\psi(\gamma)=\gamma]$, or, $\forall \gamma \supseteq \lambda[c p(\psi, \gamma) \in$ $\operatorname{subgroup}(\Delta, \gamma) \backslash\{I d\}]$.

Define the length of $\psi$ to be the least such $l$ over all factorizations of $\psi$.

(2) Suppose $\psi_{1}, \psi_{2} \in \mathcal{G}$ and $\lambda \in \Lambda$ such that

$$
\operatorname{height}(\lambda) \geq \max \left\{\text { length }\left(\psi_{1}\right), \text { length }\left(\psi_{2}\right)\right\}, \quad \text { and } \quad \psi_{1}\left|\operatorname{ext}(\lambda) \neq \psi_{2}\right| \operatorname{ext}(\lambda) .
$$

Then $\forall \gamma \supseteq \lambda, c p\left(\psi_{1}, \gamma\right) \neq c p\left(\psi_{2}, \gamma\right)$.

Proof of Fact 2. Let $\psi \in \mathcal{G}$ and $\lambda \in \Lambda$ such that height $(\lambda)=\operatorname{length}(\psi)$. Then either $\psi \mid \operatorname{ext}(\lambda)$ is the identity, or there is a unique choice of $n \in \omega$ and $i:[0, n] \rightarrow$ $E_{1}$ such that $\psi\left|\operatorname{ext}(\lambda)=\left(\varphi_{i_{0}} \circ \cdots \circ \varphi_{i_{n}}\right)\right| \operatorname{ext}(\lambda)$, and $\forall m<n\left[i_{m} \neq i_{m+1}\right]$, and $\forall m \leq n, \lambda$ is a proper extension of the first component of $\varepsilon\left(i_{m}\right)$. Suppose $\gamma \in \Lambda$ such that $\gamma \supseteq \lambda$. Then $c p(\psi, \gamma)=c p\left(\varphi_{i_{0}} \circ \cdots \circ \varphi_{i_{n}}, \gamma\right)=\delta_{\mu_{0}} \circ \cdots \circ \delta_{\mu_{n}}$, where $\forall m \leq n, \mu_{m}=\left\langle i_{m}, \gamma\right\rangle$. By Clause (1) of Fact 1, the above (standard) representation for $\psi \mid \operatorname{ext}(\lambda)$ is unique.

Clauses (1) and (2) follow from the preceding observation and Clause (1) of Fact 1 .

4.3. Homeomorphism group on the plane. We now use $\mathcal{G}$ to give a method for enlarging a given subset of $\mathcal{P}_{1}$ to a subset of $\mathcal{P}_{1}$ with a homogeneous square. 
Definition: Homeomorphism group. Let $\tilde{\mathcal{G}}=\{\tilde{\psi}: \psi \in \mathcal{G}\}$. Then $\langle\tilde{\mathcal{G}}, \circ\rangle$ is a group of homeomorphisms on $\mathcal{P}_{2}$ with $|\tilde{\mathcal{G}}|=\omega$.

Definition: $\mathcal{G}$ closure. For every $X \subseteq \mathcal{P}_{1}$ with $\mathcal{D} \subseteq X$, define the $\mathcal{G}$ closure of $X$ by $C l_{\mathcal{G}}(X)=\bigcap\left\{Y \subseteq \mathcal{P}_{1}: X \subseteq Y \& \forall \psi \in \mathcal{G}(\tilde{\psi}(\ulcorner Y \times Y\urcorner) \subseteq\ulcorner Y \times Y\urcorner)\right\}$. Note that since $\mathcal{G}$ is a group, our condition implies that for all $\psi \in \mathcal{G}(\tilde{\psi}(\ulcorner Y \times Y\urcorner)=\ulcorner Y \times Y\urcorner)$. We now have that if $Z=C l_{\mathcal{G}}(X)$, then $\forall \psi \in \mathcal{G}, \tilde{\psi}(\ulcorner Z \times Z\urcorner)=\ulcorner Z \times Z\urcorner$.

Fact 3. Since $|\mathcal{G}|=\omega$, for every $X \subseteq \mathcal{P}_{1}$ with $\mathcal{D} \subseteq X,\left|C l_{\mathcal{G}}(X)\right|=|X|$.

Lemma 2. Suppose $X \subseteq \mathcal{P}_{1}$ with $\mathcal{D} \subseteq X$. Then $S=\left\ulcorner C l_{\mathcal{G}}(X) \times C l_{\mathcal{G}}(X)\right\urcorner$ is a dense homogeneous subspace of $\mathcal{P}_{2}$.

Proof of Lemma 2. Let $f, g \in S$ with $f \neq g$. Recursively construct sequences $i: \omega \rightarrow \omega$ and $j: \omega \rightarrow \omega$ as follows. Let $i(0)=j(0)=\varepsilon^{-1}(\phi, f(0), g(0))$. Suppose $n \in \omega$, and each of $i \mid[0, n)$ and $j \mid[0, n)$ has been constructed so that

$$
\left(\varphi_{j(n-1)} \circ \cdots \circ \varphi_{j(0)}\right)(f \mid[0, n))=g \mid[0, n),
$$

and

$$
\left(\varphi_{i(n-1)} \circ \cdots \circ \varphi_{i(0)}\right)(g \mid[0, n))=f \mid[0, n) .
$$

Let $i(n)=\varepsilon^{-1}(f \mid[0, n), s, t)$ where $s=f(n)$, and $t$ is the value of the function $c p\left(\varphi_{i(n-1)} \circ \cdots \circ \varphi_{i(0)}, g \mid[0, n)\right)$ at the argument $g(n)$. Let $j(n)=\varepsilon^{-1}(g \mid[0, n), s, t)$ where $s$ is the value of the function $c p\left(\varphi_{j(n-1)} \circ \cdots \circ \varphi_{j(0)}, f \mid[0, n)\right)$ at the argument $f(n)$, and $t=g(n)$. Let $n_{0} \in \omega$ be the least point for which $f\left(n_{0}\right) \neq g\left(n_{0}\right)$. For all $n \in \omega$, let $k(2 n)=i\left(n+n_{0}\right)$ and $k(2 n+1)=j\left(n+n_{0}+1\right)$. For all $n \in \omega$, let $\psi_{n}=\varphi_{k(n)} \circ \cdots \circ \varphi_{k(0)}$.

Let $H: \mathcal{P}_{2} \rightarrow \mathcal{P}_{2}$ be defined by $H(h)=\lim _{n \rightarrow \infty} \tilde{\psi}_{n}(h)$ (pointwise convergence).

Then $H$ is a homeomorphism of $\mathcal{P}_{2}$ onto $\mathcal{P}_{2}$ that interchanges $f$ and $g$. Since $\left\langle\tilde{\psi}_{n}(h): n \in \omega\right\rangle$ is eventually constant whenever $h \notin\{f, g\}, H[S]=S$.

\section{ExPANDing AND CLOSING FINITE SUbSETS OF $\boldsymbol{\omega}$}

5.1. Expansion. Suppose $M \subseteq \omega$ is finite. Then the first and second expansions of $M$, denoted by $\exp _{1}(M)$ and $\exp _{2}(M)$ respectively, are defined from three recursively constructed auxiliary sequences.

For all $t \in \omega^{2}$, define the diagonal projections by $d \pi_{1}(t)=\left\langle\pi_{1}(t), \pi_{1}(t)\right\rangle$ if $\pi_{1}(t) \in E_{0}$ and $d \pi_{1}(t)=\langle n, n+1\rangle$ if $n \in E_{1}$ and $\pi_{1}(t) \in\{n, n+1\}$, and $d \pi_{2}(t)=$ $\left\langle\pi_{2}(t), \pi_{2}(t)\right\rangle$ if $\pi_{2}(t) \in E_{0}$ and $d \pi_{2}(t)=\langle n, n+1\rangle$ if $n \in E_{1}$ and $\pi_{2}(t) \in\{n, n+1\}$. For a finite and nonempty $T \subseteq \omega^{2}$ let $\operatorname{intmax}(T)=\max \left(\pi_{1}[T] \cup \pi_{2}[T]\right)$.

Let $A_{0}=\left\{t \in R: \pi_{1}(t) \in M\right.$, or, $\left.\pi_{2}(t) \in M\right\} \cup\left\{\langle n, n\rangle: n \in M \cap E_{0}\right\}$. Note that $\left|A_{0}\right| \leq|M|$. Suppose $k \in \omega$ and $\forall j \leq k, A_{j}$ has been constructed.

Define

$$
\begin{gathered}
B_{k}=\left\{r t(t): t \in A_{k}\right\} \backslash\left\{r t(t): \exists j<k\left[t \in A_{j}\right]\right\} \\
A_{k+1}^{\prime}=\left\{r \in R \backslash \bigcup\left\{A_{j}: j \leq k\right\}: \exists s, t \in \bigcup\left\{B_{j}: j \leq k\right\} \exists i, j \in\{1,2\}\right.
\end{gathered}
$$

such that

$$
\left(d \pi_{1}(t) \in A_{0} \& d \pi_{2}(t)=r\right) \quad \text { or } \quad\left(d \pi_{1}(t)=r \& d \pi_{2}(t) \in A_{0}\right)
$$


or

$$
\begin{gathered}
\left.\left([s \neq t \text { or } i \neq j] \&\left[r=d \pi_{i}(s)=d \pi_{j}(t)\right]\right)\right\} ; \\
C_{k}=\left\{t \in \bigcup\left\{B_{j}: j \leq k\right\}:\left\{d \pi_{1}(t), d \pi_{2}(t)\right\}\right. \\
\left.\subseteq R \backslash\left(\left(\bigcup\left\{A_{j}: j \leq k\right\}\right) \cup A_{k+1}^{\prime}\right)\right\} .
\end{gathered}
$$

If $C_{k}=\phi$, then let $A_{k+1}=A_{k+1}^{\prime}$. If $C_{k} \neq \phi$, then define $m_{k}=\operatorname{intmax}\left(d \pi_{1}\left[C_{k}\right]\right.$ $\left.\cup d \pi_{2}\left[C_{k}\right]\right)$. If $m_{k}$ exists, and either $A_{k+1}^{\prime}=\phi$ or $\operatorname{intmax}\left(A_{k+1}^{\prime}\right)<m_{k}$, then define $u_{k}, p_{k}$, and $q_{k}$ as follows; otherwise, each of these values is undefined. Let $u_{k}=\left\langle m_{k-1}, m_{k}\right\rangle$, and let $p_{k}$ be the unique (by the third disjunct of $A_{k+1}^{\prime}$ ) point in $C_{k}$ such that $d \pi_{1}\left(p_{k}\right)=u_{k}$ or $d \pi_{2}\left(p_{k}\right)=u_{k}$. Let $q_{k} \in R$ be the diagonal projection of $p_{k}$ that is not $u_{k}$ (the diagonal projections of $p_{k}$ are distinct by the third disjunct of $\left.A_{k+1}^{\prime}\right)$.

Define

$A_{k+1}=A_{k+1}^{\prime} \cup\left\{q_{k}\right\}$ if $m_{k}$ is defined and either $A_{k+1}^{\prime}=\phi$ or $\operatorname{intmax}\left(A_{k+1}^{\prime}\right)<m_{k}$, and otherwise,

$$
A_{k+1}=A_{k+1}^{\prime}
$$

Note that $A_{j} \cap A_{k}=\phi$ and $B_{j} \cap B_{k}=\phi$ whenever $j \neq k$, and $\forall k>0, A_{k} \subseteq R$.

If $A_{0} \neq \phi$, then define $a_{0}=\operatorname{intmax}\left(A_{0}\right)$. Suppose $k \in \omega$. If $A_{k+1} \neq \phi$ and $C_{k}=\phi$, then define $a_{k+1}=\operatorname{intmax}\left(A_{k+1}\right)$. If $A_{k+1} \neq \phi$ and $C_{k} \neq \phi$, then define $a_{k+1}=\max \left\{\operatorname{intmax}\left(A_{k+1}\right), m_{k}\right\}$; in this case, each of the following conditions is satisfied:

(1) intmax $\left(A_{k+1}\right)$ and $m_{k}$ are distinct integers in $E_{2}$;

(2) $a_{k+1}=m_{k}$ iff $u_{k}$ is defined;

(3) $\max \left\{\operatorname{intmax}\left(A_{k+1}\right), m_{k}\right\}=\max \left\{\operatorname{intmax}\left(A_{k+1}^{\prime}\right), m_{k}\right\}$ if $A_{k+1}^{\prime} \neq \phi$;

(4) if $A_{k+1}^{\prime}=\phi$, then $a_{k+1}=m_{k}$.

Fact 4. With the above notation, if $k \in \omega$ and each of $A_{k}$ and $A_{k+1}$ is nonempty, then $a_{k+1}<a_{k}$.

Proof of Fact 4. Note that each of the following conditions is satisfied.

(1) If $t \in \omega^{2}$ with $r t(t) \neq t$, then $r t(t) \prec t$ (see Section 4.1); therefore, $\operatorname{intmax}\left(\left\{r: \exists t \in B_{k} \backslash A_{k}\left[r=d \pi_{1}(t)\right.\right.\right.$ or $\left.\left.\left.r=d \pi_{2}(t)\right]\right\}\right) \leq \operatorname{intmax}\left(A_{k}\right)$ (if $B_{k} \backslash A_{k} \neq$ $\phi)$.

(2) Each point in $A_{k+1}^{\prime}$ is a diagonal projection of some point in $B_{k} \backslash A_{k}$.

(3) $C_{0} \subseteq B_{0} \backslash A_{0}$; if $k>0$, then $C_{k} \backslash C_{k-1} \subseteq B_{k} \backslash A_{k}$.

(4) $A_{k}$ and $A_{k+1}$ are disjoint subsets of $R \cup\left\{\langle n, n\rangle: n \in E_{0}\right\}$; so intmax $\left(A_{k}\right) \neq$ $\operatorname{intmax}\left(A_{k+1}\right)$.

(5) $A_{k}$ and $d \pi_{1}\left[C_{k}\right] \cup d \pi_{2}\left[C_{k}\right]$ are disjoint subsets of $R \cup\left\{\langle n, n\rangle: n \in E_{0}\right\}$; so $\operatorname{intmax}\left(A_{k}\right) \neq m_{k}$.

(6) Either $p_{k-1}$ is undefined, or $p_{k-1} \notin C_{k}$.

By (1), (2), and (3), $a_{k+1} \leq a_{k}$. If $a_{k}=\operatorname{intmax}\left(A_{k}\right)$, then $a_{k+1} \neq a_{k}$ by (4) and $(5)$; if $a_{k}=m_{k-1}$, then $a_{k+1} \neq a_{k}$ by $(6)$.

Definition: Number of stages. By Fact 4, we can define $l=\min \left\{k \in \omega: A_{k}=\right.$ $\phi\}$. Note that for all $j, k \in \omega$ with $j<k\left[A_{j}=\phi \Rightarrow A_{k}=\phi\right]$, and for all $k \in \omega$ $\left[A_{k+1}=\phi \Rightarrow C_{k}=\phi\right]$; in particular, $C_{l-1}=\phi$.

Corollary to Fact 4. For all $j<l$, if $u_{j}$ is defined, then $u_{j} \in R \backslash \bigcup\left\{A_{k}: k<l\right\}$, and $p_{j}$ is the unique point in $\bigcup\left\{B_{k}: k<l\right\}$ such that $u_{j}$ is a diagonal projection of $p_{j}$. 
Proof of the Corollary. By the definitions of the auxiliary sequences, $u_{j} \in R \backslash$ $\bigcup\left\{A_{k}: k \leq j+1\right\}$; and by Fact $4, u_{j} \notin \bigcup\left\{A_{k}: j+1<k<l\right\}$. Our conclusion on $p_{j}$ follows from our conclusion on $u_{j}$ and the third disjunct of $A_{k+1}^{\prime}(k<l)$.

Definition: First and second expansions. Define the first and second expansions by

(i) $\exp _{1}(M)=\pi_{1}[T] \cup \pi_{2}[T]$, where $T=\bigcup\left\{A_{k}: k<l\right\}$;

(ii) $\exp _{2}(M)=\pi_{1}[T] \cup \pi_{2}[T]$, where $T=\left(\bigcup\left\{A_{k}: k<l\right\}\right) \cup\left(\bigcup\left\{B_{k}: k<l\right\}\right)$. Note that $M \subseteq \exp _{1}(M)$ by the choice of $A_{0}$.

As auxiliary sequences in the construction of the first and second expansions, we will refer to $\left\langle A_{k}: k<l\right\rangle$ as the primary sequence and to $\left\langle B_{k}: k<l\right\rangle$ as the secondary sequence.

5.2. Canonical map. For $t \in \omega^{2}$, define the sides of $t$ to be the ordered pairs $\left\langle\pi_{1}(t), t\right\rangle$ and $\left\langle t, \pi_{2}(t)\right\rangle$ (where we picture $\omega^{2}$ as a collection of rectangles constructed from $\omega$-many vertical lines and $\omega$-many horizontal lines). For all $T \subseteq \omega^{2}$, let $s d(T)=\left\{\left\langle\pi_{1}(t), t\right\rangle: t \in T\right\} \cup\left\{\left\langle t, \pi_{2}(t)\right\rangle: t \in T\right\}$.

With the notation from the definition for expansion, the canonical map is a function from a subset of $\operatorname{sd}\left(\bigcup\left\{B_{k}: k<l\right\}\right)$ onto $\bigcup\left\{A_{k}: 0<k<l\right\}$ defined by the following rules. Suppose $r \in \bigcup\left\{A_{k}: 0<k<l\right\}$ and $t \in \bigcup\left\{B_{k}: k<l\right\}$. Then, under the canonical map:

(i) $\left\langle\pi_{1}(t), t\right\rangle \mapsto r$ iff $d \pi_{1}(t)=r$ or $\left(d \pi_{1}(t) \in A_{0} \& d \pi_{2}(t)=r\right)$ or $\left(d \pi_{1}(t) \in\left\{u_{k}\right.\right.$ : $\left.k<l\} \& d \pi_{2}(t)=r\right)$

(ii) $\left\langle t, \pi_{2}(t)\right\rangle \mapsto r$ iff $d \pi_{2}(t)=r$ or $\left(d \pi_{2}(t) \in A_{0} \& d \pi_{1}(t)=r\right)$ or $\left(d \pi_{2}(t) \in\left\{u_{k}\right.\right.$ : $\left.k<l\} \& d \pi_{1}(t)=r\right)$.

By the definition of the primary sequence and the Corollary to Fact 4, the canonical map is a well-defined single-valued function that is uniformly at least two-to-one through stage $k$ for each $k<l$; by this we mean that for each $k<l$, the inverse image of each point in $A_{k+1}$ contains at least two sides in $s d\left(\bigcup\left\{B_{j}: j \leq k\right\}\right)$. If a side of some point in some $B_{j}$ is unassigned by the rules given above, then that particular side does not belong to the domain of the canonical map.

For each $k<l$, define

$$
\begin{aligned}
& D_{k}=\left\{t \in \bigcup\left\{B_{j}: j \leq k\right\}: t \notin\left\{p_{j}: j \leq k\right\}\right. \\
& \left.\quad \& \text { at least one of } d \pi_{1}(t) \text { and } d \pi_{2}(t) \text { belongs to } R \backslash \bigcup\left\{A_{j}: j \leq k+1\right\}\right\} ; \\
& V_{k}=\left\{\left\langle\pi_{1}(t), t\right\rangle: t \in D_{k} \& d \pi_{1}(t) \in R \backslash \bigcup\left\{A_{j}: j \leq k+1\right\}\right\} \\
& \cup\left\{\left\langle t, \pi_{2}(t)\right\rangle: t \in D_{k} \& d \pi_{2}(t) \in R \backslash \bigcup\left\{A_{j}: j \leq k+1\right\}\right\} ; \\
& W_{k}=\left\{\left\langle\pi_{1}(t), t\right\rangle: t \in \bigcup\left\{B_{j}: j \leq k\right\} \& \pi_{1}(t) \in E_{0} \backslash M\right\} \\
& \cup\left\{\left\langle t, \pi_{2}(t)\right\rangle: t \in \bigcup\left\{B_{j}: j \leq k\right\} \& \pi_{2}(t) \in E_{0} \backslash M\right\} \\
& \cup \operatorname{sd}\left(\left\{t \in \bigcup\left\{B_{j}: j \leq k\right\}:\left\{\pi_{1}(t), \pi_{2}(t)\right\} \subseteq E_{0} \cup \pi_{1}\left[A_{0}\right] \cup \pi_{2}\left[A_{0}\right]\right\}\right) .
\end{aligned}
$$

A side of a point in some $B_{j}$ is defined to be unused iff it does not belong to the domain of the canonical map; we claim (proved below) that each side in $W_{l-1}$ is unused. For each $k<l$, a side in $\operatorname{sd}\left(\bigcup\left\{B_{j}: j \leq k\right\}\right)$ is used through stage $k$ iff it belongs to the inverse image under the canonical map of $\bigcup\left\{A_{j}: 0<j \leq\right.$ $k+1\}$; we claim (proved below) that $V_{k} \cup W_{k}$ consists precisely of those sides in $s d\left(\bigcup\left\{B_{j}: j \leq k\right\}\right)$ that are unused through stage $k$, and that $V_{k}$ consists precisely 
of those sides that have a potential use (that may or may not be realized) at some later stage.

To prove these claims, we will consider sides of the form $\left\langle\pi_{1}(t), t\right\rangle$ (an analogous argument handles sides of the form $\left.\left\langle t, \pi_{2}(t)\right\rangle\right)$. We first show that $V_{k}, W_{k}$, and the set of all sides that are used through stage $k$ are three mutually exclusive sets.

A side $\left\langle\pi_{1}(t), t\right\rangle$ cannot be both a member of $V_{k}$ and used through stage $k$, since in part (i) of the definition of Canonical Map, each of the first two disjuncts is contradicted by $d \pi_{1}(t) \in R \backslash \bigcup\left\{A_{j}: j \leq k+1\right\}$, and the third disjunct is contradicted by $t \notin\left\{p_{j}: j \leq k\right\}$ (see the corollary to Fact 4; we are also using the fact that if the canonical map takes $\left\langle\pi_{1}(t), t\right\rangle$ to $r \in \bigcup\left\{A_{j}: 0<j \leq k+1\right\}$ by way of the third disjunct of (i), then $\left.d \pi_{1}(t) \in\left\{u_{j}: j \leq k\right\}\right)$.

A side $\left\langle\pi_{1}(t), t\right\rangle$ cannot be both a member of $W_{k}$ and used (at any stage) by the canonical map since the latter implies:

(1) $d \pi_{1}(t) \in R \cup\{\langle n, n\rangle: n \in M\}$ (which contradicts $\pi_{1}(t) \in E_{0} \backslash M$ );

(2) at least one of the diagonal projections of $t$ belongs to $\bigcup\left\{A_{j}: 0<j<l\right\}$ (which contradicts $\left\{\pi_{1}(t), \pi_{2}(t)\right\} \subseteq E_{0} \cup \pi_{1}\left[A_{0}\right] \cup \pi_{2}\left[A_{0}\right]$ ).

Finally, $V_{k}$ and $W_{k}$ are disjoint since $d \pi_{1}(t) \in R \backslash A_{0}$ iff $\pi_{1}(t) \notin E_{0} \cup \pi_{1}\left[A_{0}\right] \cup$ $\pi_{2}\left[A_{0}\right]$.

To show that we have a cover for $s d\left(\bigcup\left\{B_{j}: j \leq k\right\}\right.$, we consider cases for a side $\left\langle\pi_{1}(t), t\right\rangle$ where $t \in \bigcup\left\{B_{j}: j \leq k\right\}$ :

(1) if $\pi_{1}(t) \in \pi_{1}\left[A_{0}\right] \cup \pi_{2}\left[A_{0}\right]$ and $\pi_{2}(t) \in E_{0} \cup \pi_{1}\left[A_{0}\right] \cup \pi_{2}\left[A_{0}\right]$, then $\left\langle\pi_{1}(t), t\right\rangle$ is unused and appears in the third term of the union defining $W_{k}$;

(2) if $\pi_{1}(t) \in \pi_{1}\left[A_{0}\right] \cup \pi_{2}\left[A_{0}\right]$ and $\pi_{2}(t) \notin E_{0} \cup \pi_{1}\left[A_{0}\right] \cup \pi_{2}\left[A_{0}\right]$, then $d \pi_{1}(t) \in A_{0}$ and $d \pi_{2}(t) \in R \backslash A_{0}$, and therefore, $\left\langle\pi_{1}(t), t\right\rangle$ is used since $d \pi_{2}(t) \in \bigcup\left\{A_{j}: j \leq k+1\right\}$ by the first disjunct of $A_{j+1}^{\prime}(j \leq k)$;

(3) if $\pi_{1}(t) \in E_{0} \backslash M$, then $\left\langle\pi_{1}(t), t\right\rangle$ is unused and appears in the first term of the union defining $W_{k}$;

(4) if $t=p_{j}$ for some $j \leq k$, then $\left\langle\pi_{1}(t), t\right\rangle$ is used and mapped to $q_{j}$;

(5) if $d \pi_{1}(t) \in R \backslash A_{0}$ and $t \notin\left\{p_{j}: j \leq k\right\}$, then $\left\langle\pi_{1}(t), t\right\rangle$ is used by virtue of being mapped to $d \pi_{1}(t) \in \bigcup\left\{A_{j}: j \leq k+1\right\}$, or $\left\langle\pi_{1}(t), t\right\rangle$ is unused through stage $k$ by virtue of $d \pi_{1}(t) \notin \bigcup\left\{A_{j}: j \leq k+1\right\}$, and appears in $V_{k}$.

5.3. Intersection of the second expansion with $\boldsymbol{E}_{\mathbf{0}}$. We now introduce at the level of $\omega$ the analogue of the $\mathcal{G}$ closure operator.

Definition: Expanded $\Delta$ closure. Suppose that $M \subseteq \omega$ is finite. Then the expanded $\Delta$ closure of $M$, denoted $C l_{\Delta}(M)$, is defined by $C l_{\Delta}(M)=\phi$ if $M=\phi$, and otherwise, by $C l_{\Delta}(M)=\bigcap\left\{L \subseteq \omega: \exp _{2}(M) \subseteq L \& \forall \eta \in \Delta\left(\eta\left[L^{2}\right] \subseteq L^{2}\right)\right\}$ ( since $\Delta$ is a group, our condition implies that $\forall \eta \in \Delta\left(\eta\left[L^{2}\right]=L^{2}\right)$ ).

Proposition 1. Suppose $M \subseteq \omega$ is finite, and $L=C l_{\Delta}(M)$. Then

(1) $\left|\exp _{2}(M) \cap E_{0}\right| \leq 2|M|$;

(2) $L \subseteq \exp _{2}(M) \cup E_{1} \cup E_{2}$.

Proof of Proposition 1. See 5.1 and 5.2 for the definitions of the auxiliary sequences $A, B, V$, and $W$, and the integer $l \in \omega$.

Clause (1). Note that $\left|\exp _{2}(M) \cap E_{0}\right| \leq\left|W_{l-1}\right|$ (recall that $\{\langle n, n\rangle: n \in$ $\left.E_{0} \cap M\right\} \subseteq B_{0}$; it then follows that the function sending $\left\langle\pi_{1}(t), t\right\rangle$ to $\pi_{1}(t)$, and sending $\left\langle t, \pi_{2}(t)\right\rangle$ to $\pi_{2}(t)$ maps $W_{l-1}$ onto $\left.\exp _{2}(M) \cap E_{0}\right)$. Therefore, Clause (1) follows immediately from the claim below, and $\left|A_{0}\right| \leq|M|$. 
Claim. $\forall k<l, 2\left|A_{k+1}\right|+\left|V_{k}\right|+\left|W_{k}\right| \leq 2\left|A_{0}\right|$.

Proof of the Claim. First, recall that $\forall k<l, V_{k}$ and $W_{k}$ are disjoint subsets of those sides in $s d\left(\bigcup\left\{B_{j}: j \leq k\right\}\right)$ that are unused through stage $k$.

We now prove the claim by induction on $k$. For $k=0$,

$$
2\left|A_{1}\right|+\left|V_{0}\right|+\left|W_{0}\right| \leq\left|\operatorname{sd}\left(B_{0}\right)\right| \leq 2\left|A_{0}\right|
$$

by the note above and the fact that the canonical map is at least two-to-one through stage zero.

For the Induction Hypothesis, suppose $k<l$, and

$$
2\left|A_{k+1}\right|+\left|V_{k}\right|+\left|W_{k}\right| \leq 2\left|A_{0}\right| .
$$

Since the canonical map is at least two-to-one through stage $k+1,2\left|A_{k+2}\right|$ is bounded above by the cardinality of those sides in $s d\left(B_{k+1}\right) \cup V_{k}$ that are used at stage $k+1$. By the note above, $V_{k+1}$ and $W_{k+1} \backslash W_{k}$ are disjoint subsets of those sides in $s d\left(B_{k+1}\right) \cup V_{k}$ that are unused through stage $k+1$. Also, $B_{k+1}$ and $D_{k}$ are disjoint; so $s d\left(B_{k+1}\right)$ and $V_{k}$ are disjoint. Therefore,

$$
\begin{aligned}
2\left|A_{k+2}\right|+\mid & V_{k+1}|+| W_{k+1} \backslash W_{k} \mid \\
& \leq\left|s d\left(B_{k+1}\right)\right|+\left|V_{k}\right| \\
& \leq 2\left|A_{k+1}\right|+\left|V_{k}\right| .
\end{aligned}
$$

Add $\left|W_{k}\right|$ to each end, and apply the Induction Hypothesis.

Clause (2). We recursively construct a sequence of nested increasing subsets of $\omega,\left\langle N_{n}: n \in \omega\right\rangle$, such that $L=\bigcup\left\{N_{n}: n \in \omega\right\}$. Let $N_{0}=\exp _{2}(M), S_{0}=$ $N_{0}^{2}$, and $T_{0}=\bigcup\left\{\operatorname{orbit}(t): t \in S_{0}\right\}$. By the definition of the secondary sequence from the primary sequence, and the third disjunct in the definition of $A_{k+1}^{\prime}(k<$ $l), r t\left[S_{0}\right] \subseteq S_{0}$; therefore, $T_{0} \subseteq S_{0} \cup R$ (Clause (3) of Fact 1). Suppose $n>0$. Then define $N_{n}=\pi_{1}\left[T_{n-1}\right] \cup \pi_{2}\left[T_{n-1}\right], S_{n}=N_{n}^{2}$, and $T_{n}=\bigcup\left\{\operatorname{orbit}(t): t \in S_{n}\right\}$. For the Recursion Hypothesis, suppose that $\operatorname{rt}\left[S_{n-1}\right] \subseteq S_{n-1}$. This implies that $T_{n-1} \subseteq S_{n-1} \cup R$, which, in turn, implies that $N_{n} \subseteq N_{n-1} \cup E_{1} \cup E_{2}$. Note the following fact on projections: Suppose $s, t \in T_{n-1}, r \in S_{n}$, and $i, j \in\{1,2\}$ such that $r=\left\langle\pi_{i}(s), \pi_{j}(t)\right\rangle$. Suppose further that $s \in R$ (respectively, $t \in R$ ). Then $r \in R$ iff $r=s$ (respectively, $r=t$ ). This fact in combination with the fact that $S_{n-1}$ is a square for which $S_{n-1} \subseteq T_{n-1} \subseteq S_{n-1} \cup R$ implies that $S_{n} \subseteq T_{n-1} \cup\left(\omega^{2} \backslash R\right)$. We now have $T_{n-1} \subseteq S_{n} \subseteq T_{n-1} \cup\left(\omega_{2} \backslash R\right)$; and, since $T_{n-1}$ is a union of $\Delta$ orbits, $r t\left[T_{n-1}\right] \subseteq T_{n-1}$. These two results yield $r t\left[S_{n}\right] \subseteq S_{n}$, and so the Recursion Hypothesis is preserved.

\section{SPECial OPERATORS ON $\boldsymbol{\omega}$}

We need to define an operator $\theta$ at two levels. The first level concerns arguments (in part) and values that are functions from $\omega$ into $\omega$; at the second level (defined in Section 7), $\omega$ is replaced by open subsets of $\mathcal{P}_{1}$.

Definition: Standard composition on $\omega$. An argument for $\theta$ (at the level of $\omega$ ) is a four-tuple $\langle i, \eta, f, g\rangle$ such that $i \in\{1,2\}, \eta \in \Delta$, and $f, g: \omega \rightarrow \omega$. We define $\theta(i, \eta, f, g)=h$ where $h: \omega \rightarrow \omega$ such that $\forall n \in \omega, h(n)=\pi_{i}(\eta(f(n), g(n)))$.

Suppose $M \subseteq \omega$. The ground set determined by $M$, denoted $g s(M)$, is defined by $g s(M)=\{f: \omega \rightarrow \omega \mid f$ is the identity function on $\omega$, or $f$ is constant with range $\{m\}$ where $m \in M\}$ (if $M=\phi$, then $g s(M)$ contains only the identity function; and 
if $M=\omega$, then $g s(M)$ contains the identity and all constant functions). The set of all standard compositions on $\omega$ determined by $M$, denoted $s c(M)$, is the collection generated from $g s(M)$ by repeated applications of $\theta: h \in s c(M)$ iff $h \in g s(M)$, or $\exists i \in\{1,2\} \exists \eta \in \Delta \exists f, g \in s c(M)$ with at least one of $f$ and $g$ nonconstant such that $h=\theta(i, \eta, f, g)$. The set of all special operators on $\omega$ determined by $M$, denoted $\mathcal{O}(M)$, is defined by $\mathcal{O}(M)=\{f \in s c(M): f$ is neither the identity nor constant $\}$; so $\mathcal{O}(M)$ and $g s(M)$ are disjoint (we prove below that $\mathcal{O}(M)=s c(M) \backslash g s(M)$ ).

Now we define the level of a standard composition over $\omega$ by the following recursion. If $h \in g s(\omega)$, then $\operatorname{lev}(h)=0$. Suppose $n \in \omega$ and $h \in s c(\omega)$. Suppose that each level with an index less than or equal to $n$ has been defined. Then we define $\operatorname{lev}(h)=n+1$ if $h \notin\{f \in s c(\omega): \operatorname{lev}(f) \leq n\}$, and $\exists\langle i, \eta, f, g\rangle$ such that $i \in\{1,2\}, \eta \in \Delta, f, g \in \operatorname{sc}(M)$, the level of each of $f$ and $g$ is less than or equal to $n$, at least one of $f$ and $g$ is nonconstant, and $h=\theta(i, \eta, f, g)$; in this case, $\langle i, \eta, f, g\rangle$ is defined to be a minimal $\theta$ argument for $h$. Each $h \in s c(\omega) \backslash g s(\omega)$ has at least one minimal argument by the recursive construction of $s c(M)$; uniqueness is proved below. If $\langle i, \eta, f, g\rangle$ is minimal, then $\left\langle h_{1}, h_{2}\right\rangle$ is defined to be a redundant ordered pair (and each of $h_{1}$ and $h_{2}$ is defined to be a companion of the other) in case $h_{1}=\theta(1, \eta, f, g)$ and $h_{2}=\theta(2, \eta, f, g)$; note that the order is relevant, and that the adjective refers to constructing arguments for $\theta$ (as we will see below). Also note that each $h \in s c(\omega) \backslash g s(\omega)$ has a unique companion if minimal $\theta$ arguments are unique.

Proposition 2. Suppose that $M \subseteq \omega$ is finite, and let $L=C l_{\Delta}(M)$. Then

(1) $\forall f \in s c(M) \forall n \in L[f(n) \in L]$;

(2) $\mathcal{O}(M)=s c(M) \backslash g s(M)$ (i.e., $s c(M) \backslash g s(M)$ is devoid of constant functions);

(3) $\forall f \in \mathcal{O}(M) \forall n \in \omega \backslash L\left[f(n) \in\left(E_{1} \cup E_{2}\right) \backslash\left(\exp _{1}(M) \cup\{m \in \omega: m \leq n\}\right)\right]$;

(4) $\forall f \in \mathcal{O}(M)[f \mid \omega \backslash L$ is one-to-one $]$;

(5) $\forall f, g \in s c(M)$ with $f \neq g \forall n \in \omega \backslash L[f(n) \neq g(n)]$;

(6) $\forall f, g \in s c(M)$ where $\langle f, g\rangle$ is not redundant and at least one of $f$ and $g$ is nonconstant, and $\forall n \in \omega \backslash L\left[\langle f(n), g(n)\rangle \in \omega^{2} \backslash R\right]$;

(7) $\forall f, g \in s c(M)$, with at least one of $f$ and $g$ nonconstant, the function $n \mapsto$ $\langle f(n), g(n)\rangle$ is one-to-one on $\omega \backslash L$;

(8) $\forall n \in \omega \backslash L$, the function $\langle f, g\rangle \mapsto\langle f(n), g(n)\rangle$ is one-to-one on $\{\langle f, g\rangle: f, g \in$ $s c(M)\}$.

Proof of Proposition 2. The proof is by induction on the complexity of standard compositions (with the exception of the first clause which follows immediately from $M \subseteq L$ and the definition of the expanded $\Delta$-closure).

Let $l \in \omega,\left\langle A_{k}: k<l\right\rangle$, and $\left\langle B_{k}: k<l\right\rangle$ denote the auxiliary concepts used in the definitions of the first and second expansions of $M$, where $A$ is primary and $B$ is secondary, and $l$ is the bound on the number of stages.

We begin with two preliminary observations:

(i) Clause $(4) \Rightarrow$ Clause (7) (by definition, $\mathcal{O}(M)$ contains every nonconstant function in $s c(M)$ other than the identity);

(ii) Clause (5) $\Rightarrow$ Clause (8).

Ground step for the induction. We need to establish Clauses (5) and (6) with $g s(M)$ in place of $s c(M)$.

Clause (5). Either we have two distinct constant functions; or, one function is the identity, and the other is constant with a value that lies in $M \subseteq L$. 
Clause (6). If $f=g=$ Identity on $\omega$, then we use the fact that $R$ is disjoint from the diagonal of $\omega^{2}$. If one of the functions is constant (in which case, its value lies in $M$ ) and the other is the identity, then our result follows from the choice of $A$, and $\exp _{1}(M) \subseteq L$.

Induction Hypothesis. Suppose $k \in \omega$ and each of Clauses (3) through (6) holds if in each case we further require that each of $\operatorname{lev}(f)$ and $\operatorname{lev}(g)$ is less than or equal to $k$. Also, suppose that $\forall f \in s c(M)$ with $l e v(f) \leq k[f$ is constant $\Rightarrow f \in g s(M)]$.

Suppose that $h=\theta(i, \eta, f, g)$ where $\langle i, \eta, f, g\rangle$ is a minimal $\theta$ argument for $h$ (so at least one of $f$ and $g$ is either the identity or a special operator); and, $\operatorname{lev}(h)=k+1$. Minimality implies that the level of $h$ exceeds that of both $f$ and $g, \eta \neq I d$, and $\langle f, g\rangle$ is not redundant (in particular, each of $l e v(f)$ and $l e v(g)$ is less than or equal to $k$ ). If $\langle f, g\rangle$ is redundant, then we can choose minimal $\theta$ arguments $\left\langle 1, \eta^{\prime}, f^{\prime}, g^{\prime}\right\rangle$ and $\left\langle 2, \eta^{\prime}, f^{\prime}, g^{\prime}\right\rangle$ for $f$ and $g$ respectively; but then $h=\theta\left(i, \eta \circ \eta^{\prime}, f^{\prime}, g^{\prime}\right)$, which implies that $l e v(h) \leq k$. We prove below that the conditions $\eta \neq I d$ and $\langle f, g\rangle$ not redundant are also sufficient for minimality.

Induction Step for Clauses (2), (3), and (4). By Clause (6) for the Induction Hypothesis, for each $n \in \omega \backslash L,\langle f(n), g(n)\rangle \in \omega^{2} \backslash R$; so by Clause (7) for the Induction Hypothesis, and Clause (3) of Fact 1, the function $n \mapsto \eta(f(n), g(n))$ maps $\omega \backslash L$ one-to-one into $R$. Therefore, $h \mid \omega \backslash L$ is one-to-one, and $h[\omega \backslash L] \subseteq$ $E_{1} \cup E_{2}$. Also, recall that for all roots $t \in \omega^{2}$ and for all $\eta \in \Delta \backslash\{I d\}, t \prec \eta(t)$. Therefore, $\forall n \in \omega \backslash L[h(n)>n]$ (we are using that $f(n) \geq n$ or $g(n) \geq n$ ). Since $h$ is neither constant nor the identity, $h \in \mathcal{O}(M) \backslash g s(M)$. This completes the Induction Step for Clauses (2) and (4) of Proposition 2. To finish for Clause (3), we need to show that $\forall n \in \omega \backslash L\left[h(n) \notin \exp _{1}(M)\right]$. This follows from the fact that $\langle f(n), g(n)\rangle \notin R$ (and is therefore a root), and from the claim below (since $\left.\bigcup\left\{B_{k}: k<l\right\}=\left\{s \in \omega^{2}: \exists k<l \exists t \in A_{k}[s=r t(t)]\right\}\right)$.

Claim. For every $n \in \omega \backslash L\left[\langle f(n), g(n)\rangle \in \omega^{2} \backslash \bigcup\left\{B_{k}: k<l\right\}\right]$.

Proof of the Claim. If either $f$ or $g$ is the identity, then our conclusion follows from $\bigcup\left\{B_{k}: k<l\right\} \subseteq L^{2}$. Fix $n \in \omega \backslash L$. Let $s=\langle f(n), g(n)\rangle$. Suppose $f \in \mathcal{O}(M)$ and $g$ is constant. By Clause (2) of Proposition 2 for the Induction Hypothesis, $g(n) \in M$. By Clause (3) of Proposition 2 for the Induction Hypothesis, $f(n) \notin \exp _{1}(M)$, but $d \pi_{1}(s) \in R$ since $f(n) \in E_{1} \cup E_{2}$. By the second disjunct in the definition of $A_{k+1}^{\prime}(k<l),\left(d \pi_{1}(s) \in R \& \pi_{2}(s) \in M \& s \in \bigcup\left\{B_{k}: k<l\right\}\right) \Rightarrow \pi_{1}(s) \in$ $\exp _{1}(M)$ (use the first disjunct if $f$ is constant and $g \in \mathcal{O}(M)$ ). Finally, take the contrapositive. Suppose $f, g \in \mathcal{O}(M)$. Then (again by the Induction Hypothesis) $\{f(n), g(n)\} \subseteq\left(E_{1} \cup E_{2}\right) \backslash \exp _{1}(M)$; therefore, $\left\{d \pi_{1}(s), d \pi_{2}(s)\right\} \subseteq R \backslash \bigcup\left\{A_{k}: k<l\right\}$. Our conclusion now follows from the definition of the extension of $A_{k+1}$ from $A_{k+1}^{\prime}$ (which implies that $\forall t \in \bigcup\left\{B_{k}: k<l\right\}$ with $\left\{d \pi_{1}(t), d \pi_{2}(t)\right\} \subseteq R$, at least one of $d \pi_{1}(t)$ and $d \pi_{2}(t)$ belongs to $\left.\bigcup\left\{A_{k}: k<l\right\}\right)$.

Induction Step for Clause (5) of Proposition 2. Suppose $h^{\prime} \in s c(M)$ with lev $\left(h^{\prime}\right) \leq k+1$. Let $n \in \omega \backslash L$. By Clause (3) of Proposition 2 for $h$ : if $h^{\prime}$ is the identity, then $h^{\prime}(n)=n<h(n)$; and, if $h^{\prime}$ is constant, then $h^{\prime}(n) \in M$ (Clause (2) of Proposition 2 for $h^{\prime}$ ), whereas $h(n) \in \omega \backslash M$ since $M \subseteq \exp _{1}(M)$. Suppose $h^{\prime} \in \mathcal{O}(M)$ and $h^{\prime}(n)=h(n)$. Let $\left\langle i^{\prime}, \eta^{\prime}, f^{\prime}, g^{\prime}\right\rangle$ be a minimal $\theta$ argument for $h^{\prime}$. By Clause (6) of Proposition 2 for the Induction Hypothesis, and Clause (3) of Fact 1, 
$\left\langle f^{\prime}(n), g^{\prime}(n)\right\rangle=\langle f(n), g(n)\rangle, \eta^{\prime}=\eta$, and $i^{\prime}=i$. By Clause (5) of Proposition 2 for the Induction Hypothesis, $f^{\prime}=f$ and $g^{\prime}=g$. Thus, $h^{\prime}=h$.

Induction Step for Clause (6) of Proposition 2. Suppose $h^{\prime} \in \operatorname{sc}(M)$ such that $l e v\left(h^{\prime}\right) \leq k+1,\left\langle h, h^{\prime}\right\rangle$ is not redundant, and $\exists n \in \omega \backslash L$ such that $\left\langle h(n), h^{\prime}(n)\right\rangle$ belongs to $R$. Then $h(n) \in E_{1}$ forcing $i=1$. Let $h_{0}$ be the companion of $h$ defined by $h_{0}=\theta(2, \eta, f, g)$. Then $h_{0}(n)=h^{\prime}(n)$. So by Clause (5) of Proposition 2 for $h_{0}$ and $h^{\prime}, h_{0}=h^{\prime}$ contradicting the hypothesis that $\left\langle h, h^{\prime}\right\rangle$ is not redundant. Note that the same argument shows that $\left\langle h^{\prime}(n), h(n)\right\rangle \in R$ implies $\left\langle h^{\prime}, h\right\rangle$ is redundant. (This last observation is needed because the hypotheses on $h$ and $h^{\prime}$ are not the same. So the interchange must be considered; in this case, $i=2$ and $h_{0}=\theta(1, \eta, f, g)$.)

Corollary 1 to Proposition 2. Suppose that $f, g \in s c(\omega)$ with at least one of $f$ and $g$ nonconstant, $i \in\{1,2\}$, and $\eta \in \Delta$. Then $\langle i, \eta, f, g\rangle$ is minimal iff $\eta \neq$ $I d$, and $\langle f, g\rangle$ is not redundant. The restriction of $\theta$ to the set of all minimal $\theta$ arguments is one-to-one and onto $\mathcal{O}(\omega)$.

Proof of Corollary 1. As noted above, arguments $\langle i, \eta, f, g\rangle$, where $\eta=I d$ or $\langle f, g\rangle$ is redundant, are not minimal. Let $\theta_{0}$ denote the restriction of $\theta$ to $\{\langle i, \eta, f, g\rangle$ : $i \in\{1,2\}, \eta \in \Delta \backslash\{I d\}, f, g \in \operatorname{sc}(\omega)$ with at least one of $f$ and $g$ nonconstant, and $\langle f, g\rangle$ is not redundant $\}$. Note that $s c(\omega)=\bigcup\{s c(M): M \subseteq \omega$ is finite ; so Proposition 2 applies to the current situation. By Clauses (6) and (7) of Proposition 2 and Clause (3) of Fact 1, values of $\theta_{0}$ are nonconstant functions different from the identity (either $f(n) \geq n$ or $g(n) \geq n$, and, $t \prec \eta(t)$ for every root $t$ ); so the range of $\theta_{0}$ is a subset of $\mathcal{O}(\omega)$. Since every value in $\mathcal{O}(\omega)$ has at least one minimal $\theta$ argument, $\operatorname{ran}\left(\theta_{0}\right)=\mathcal{O}(\omega)$. So we can establish both parts of the corollary simultaneously by showing that $\theta_{0}$ is one-to-one. This fact follows from Clauses (6) and (8) of Proposition (2), and Clause (3) of Fact 1.

Corollary 2 to Proposition 2. Suppose $M \subseteq \omega$ is finite, $L=C l_{\Delta}(M)$, and $f, g \in \mathcal{O}(M)$ such that $\langle f, g\rangle$ is redundant. Then the last step in the $\theta$ evaluation of the (unique) minimal argument for $f$ (respectively, $g$ ) is a projection to the first (respectively, second) coordinate; and, $\forall n \in \omega \backslash L,\langle f(n), g(n)\rangle \in R$.

Proof of Corollary 2. By the first corollary to Proposition 2 and the fact that $\langle f, g\rangle$ is redundant, there is a unique argument $\left\langle i, \eta, f_{0}, g_{0}\right\rangle \in \operatorname{dom}\left(\theta_{0}\right)$ such that $f=$ $\theta_{0}\left(1, \eta, f_{0}, g_{0}\right)$, and $g=\theta_{0}\left(2, \eta, f_{0}, g_{0}\right)$; the second part of the conclusion follows from the preceding observation, and, Clause (6) of Proposition 2 and Clause (3) of Fact 1 applied to $\left\langle f_{0}, g_{0}\right\rangle$.

\section{SPECIAL OPERATORS ON OPEN SUBSETS OF THE IRRATIONALS}

We now define $\theta$ at the level of $\mathcal{P}_{1}$. At this level, arguments (in part) and values of $\theta$ are functions from basic open subsets of $\mathcal{P}_{1}$ into $\mathcal{P}_{1}$ (the functions of interest are continuous).

Definition: Standard composition on an open subset of $\mathcal{P}_{1}$. An argument for $\theta$ (at the level of $\mathcal{P}_{1}$ ) is a four-tuple $\langle i, \psi, F, G\rangle$ such that $i \in\{1,2\}, \psi \in \mathcal{G}$, and for some $\sigma \in \Sigma, F, G: \mathcal{B}(\sigma) \rightarrow \mathcal{P}_{1}$. We define $\theta(i, \psi, F, G)=H$ where $H: \mathcal{B}(\sigma) \rightarrow \mathcal{P}_{1}$ such that $\forall h \in \mathcal{B}(\sigma), H(h)=\pi_{i} \circ(\tilde{\psi}(\ulcorner F(h), G(h)\urcorner))$ (note that the right-hand factor of the composition maps $\omega$ into $\omega^{2}$, and the left-hand factor maps $\omega^{2}$ into $\left.\omega\right)$. 
Suppose $X \subseteq \mathcal{P}_{1}$ is finite. Let $\operatorname{split}(X)$ denote the least $l \in \omega$ such that $\forall f, g \in X$ with $f \neq g \exists n \in \omega$ with $n<l[f(n) \neq g(n)]$. The ground set determined by $X$, denoted $g s(X)$, is defined by $g s(X)=\left\{F: \mathcal{B}(\sigma) \rightarrow \mathcal{P}_{1} \mid \sigma \in \Sigma\right.$ with height $(\sigma) \geq$ $\operatorname{split}(X)$; and, $F$ is the identity function on $\mathcal{B}(\sigma)$, or $F$ is constant with range $\{f\} \subseteq$ $X\}$. The set of all standard compositions on open subsets of $\mathcal{P}_{1}$ determined by $X$, denoted $s c(X)$, is the collection generated from $g s(X)$ by repeated applications of $\theta: H \in s c(X)$ iff $H \in g s(X)$, or, $\exists i \in\{1,2\} \exists \psi \in \mathcal{G} \exists F, G \in s c(X) \exists \sigma \in \Sigma$ such that the following four conditions are satisfied:

(i) height $(\sigma) \geq \max \{\operatorname{length}(\psi), \operatorname{split}(X)\}$,

(ii) $\operatorname{dom}(F)=\operatorname{dom}(G)=\mathcal{B}(\sigma)$,

(iii) at least one of $F$ and $G$ is nonconstant, and

(iv) $H=\theta(i, \psi, F, G)$.

The set of all special operators on open subsets of $\mathcal{P}_{1}$ determined by $X$, denoted $\mathcal{O}(X)$, is defined by $\mathcal{O}(X)=\{F \in \operatorname{sc}(X): F$ is neither an identity function nor constant\}.

Proposition 3 implies that if $F \in g s(X)$ (respectively, $\mathcal{O}(X), s c(X)$ ) and $\sigma \in \Sigma$ with $\mathcal{B}(\sigma)=\operatorname{dom}(F)$, then $\forall \tau \in \Sigma$ with $\tau \supseteq \sigma, F \mid \mathcal{B}(\tau) \in g s(X)$ (respectively, $\mathcal{O}(X), s c(X))$.

Suppose $X \subseteq \mathcal{P}_{1}$. Then we define $g s(X)$ (respectively, $\mathcal{O}(X), s c(X)$ ) by $g s(X)$ (respectively, $\mathcal{O}(X), s c(X))=\bigcup\{g s(Y)$ (respectively, $\mathcal{O}(Y), s c(Y)): Y$ is a finite subset of $X$ \}.

Recursively define the level of a standard composition over $\mathcal{P}_{1}$ as follows. If $H \in g s\left(\mathcal{P}_{1}\right)$, then define $\operatorname{lev}(H)=0$. Suppose $k \in \omega$, and each level indexed by a value less than or equal to $k$ has been defined. Suppose $H \in s c(X)$, where $X \subseteq \mathcal{P}_{1}$ is finite, such that $H$ does not belong to level $m$ for all $m \leq k$, and $\exists\langle i, \psi, F, G\rangle$ where $i \in\{1,2\}, \psi \in \mathcal{G}, F, G \in \operatorname{sc}(X)$ such that the level of each of $F$ and $G$ is less than or equal to $k$ with at least one of $F$ and $G$ nonconstant, and $H=\theta(i, \psi, F, G)$; in this case, $\operatorname{lev}(H)=k+1$, and $\langle i, \psi, F, G\rangle$ is defined to be a minimal $\theta$ argument for $H$. Existence of such an argument follows from the recursive definition of standard composition. Uniqueness of such an argument is shown below; uniqueness in the second coordinate refers to $\psi \mid \operatorname{ext}(\lambda)$ where $\lambda$ has the following definition. In the next paragraph, we show that every standard composition over $\mathcal{P}_{1}$ is induced by a unique block function. Let $\sigma \in \Sigma$ such that $\mathcal{B}(\sigma)$ is the common domain of $F$ and $G$. Let $\chi_{1}, \chi_{2}: \operatorname{ext}(\sigma) \rightarrow \Sigma$ be block functions such that $\tilde{\chi}_{1}=F$ and $\tilde{\chi}_{2}=G$. Define: $\lambda=\left\ulcorner\chi_{1}(\sigma), \chi_{2}(\sigma)\right\urcorner$. If $\langle i, \psi, F, G\rangle$ is minimal, then $\left\langle H_{1}, H_{2}\right\rangle$ is defined to be a redundant ordered pair in case $H_{1}=\theta(1, \psi, F, G)$ and $H_{2}=\theta(2, \psi, F, G)$; in this event, $H_{1}$ and $H_{2}$ are defined to be companions of one another. Note that existence of a unique companion for each member of $\mathcal{O}\left(\mathcal{P}_{1}\right)$ follows from the uniqueness of minimal $\theta$ arguments. Also note that if $\langle i, \psi, F, G\rangle$ is minimal, then the restriction of $\psi$ to $\operatorname{ext}(\lambda)$ is not the identity, and, $\langle F, G\rangle$ is not redundant (for redundancy, use the same argument that was given for compositions over $\omega)$.

Suppose $H \in \operatorname{sc}\left(\mathcal{P}_{1}\right)$ and $\sigma \in \Sigma$ such that $\operatorname{dom}(H)=\mathcal{B}(\sigma)$. Then there exists a unique block function $\chi$ such that $\tilde{\chi}=H$ and $\operatorname{dom}(\chi)=\operatorname{ext}(\sigma)$. If $H$ is the identity on $\mathcal{B}(\sigma)$, then $\chi$ is the identity on $\operatorname{ext}(\sigma)$; and, $\forall \tau \in \Sigma$ such that $\tau \supseteq \sigma, c p(\chi, \tau)$ is the identity on $\omega^{2}$. If $H$ is constant with range $\{h\}$, then $\chi(\sigma)=h \mid[0$, height $(\sigma))$, and $\forall \tau \subseteq \sigma, c p(X, \tau)$ is constant with range $\{h($ height $(\tau))\}$. Suppose $H=\theta(i, \psi, F, G)$, and $\chi_{1}$ and $\chi_{2}$ are block functions with $\tilde{\chi}_{1}=F$ and $\tilde{\chi}_{2}=G$. Then $\forall \tau \supseteq \sigma$ : $\chi(\tau)=\pi_{i} \circ(\psi(\lambda))$, where $\lambda=\left\ulcorner\chi_{1}(\tau), \chi_{2}(\tau)\right\urcorner$; and, $c p(\chi, \tau)(n)=\pi_{i}(c p(\psi, \lambda)(t))$ 
where $n \in \omega, m_{1}=c p\left(\chi_{1}, \tau\right)(n), m_{2}=c p\left(\chi_{2}, \tau\right)(n)$, and $t=\left\langle m_{1}, m_{2}\right\rangle$. Proposition 3 implies that if $\langle i, \psi, F, G\rangle$ is minimal, and $H^{\prime}$ is the companion of $H$ defined by $H^{\prime}=\theta(j, \psi, F, G)$ where $j \neq i$, and $\chi^{\prime}$ is the underlying block function for $H^{\prime}$, then $\forall \tau \supseteq \sigma, c p(\chi, \tau)$ and $c p\left(\chi^{\prime}, \tau\right)$ are companions in $\mathcal{O}(\omega)$.

Proposition 3. Suppose $X \subseteq \mathcal{P}_{1}$ is finite. Then each of the following conditions is satisfied:

(1) $\mathcal{O}(X)=s c(X) \backslash g s(X)$.

(2) Suppose $H \in \operatorname{sc}(X) \backslash g s(X)$, and $\sigma \in \Sigma$ with $\operatorname{dom}(H)=\mathcal{B}(\sigma)$, and $\chi$ is a block function with $\tilde{\chi}=H$. Then

(2.1) there is a unique minimal $\theta$ argument for $H$;

(2.2) if $\langle i, \psi, F, G\rangle$ is a minimal $\theta$ argument for $H$, then $\forall \tau \in \Sigma$ with $\tau \supseteq \sigma$, $\left\langle c p\left(\chi_{1}, \tau\right), c p\left(\chi_{2}, \tau\right)\right\rangle$ is not redundant, where $\chi_{1}$ and $\chi_{2}$ are block functions with $\tilde{\chi}_{1}=F$ and $\tilde{\chi}_{2}=G$;

(2.3) $\forall \tau \in \Sigma$ with $\tau \supseteq \sigma[c p(\chi, \tau) \in \mathcal{O}(M)$ where $M=\{h($ height $(\tau)): h \in X\}]$; and,

(2.4) $\forall \tau_{1}, \tau_{2} \in \Sigma$ where $\tau_{1}$ and $\tau_{2}$ are distinct extensions of $\sigma$ with height $\left(\tau_{1}\right)=$ height $\left(\tau_{2}\right)=m,\left\{c p\left(\chi, \tau_{1}\right)(n): n \in \omega \backslash L\right\}$ and $\left\{c p\left(\chi, \tau_{2}\right)(n): n \in \omega \backslash L\right\}$ are disjoint, where $L=C l_{\Delta}(\{h(m): h \in X\})$.

(3) Suppose $H, H^{\prime} \in s c(X) \backslash g s(X)$, and $\chi$ and $\chi^{\prime}$ are block functions with $\tilde{\chi}=H$ and $\tilde{\chi}^{\prime}=H^{\prime}$. Suppose $\sigma, \tau \in \Sigma$ such that $\tau \supseteq \sigma, \operatorname{dom}(H)=\operatorname{dom}\left(H^{\prime}\right)=\mathcal{B}(\sigma)$, and $c p(\chi, \tau)=c p\left(\chi^{\prime}, \tau\right)$. Then $H=H^{\prime}$.

Proof of Proposition 3. The proof is by induction on the complexity of standard compositions.

For the Induction Hypothesis, suppose that $k \in \omega$, and parts (2) and (3) of Proposition 3 hold whenever $\operatorname{lev}(H) \leq k$ and $\operatorname{lev}\left(H^{\prime}\right) \leq k$; and, suppose that if $\operatorname{lev}(H) \leq k$ and $H$ is constant, then $H \in g s(X)$.

Suppose that $H \in \operatorname{sc}(X)$ with $\operatorname{lev}(H)=k+1$. Suppose further that $\langle i, \psi, F, G\rangle$ is a minimal $\theta$ argument for $H$ (so $\operatorname{lev}(F) \leq k$ and $\operatorname{lev}(G) \leq k$ ). Let $\sigma \in \Sigma$ such that $\mathcal{B}(\sigma)$ is the common domain of $F, G$, and $H$. Let $\chi, \chi_{1}$, and, $\chi_{2}$ be block functions such that $H=\tilde{\chi}, F=\tilde{\chi}_{1}$, and $G=\tilde{\chi}_{2}$. Recall that minimality implies that $\langle F, G\rangle$ is not redundant, and $\psi \mid \operatorname{ext}(\lambda) \neq I d$, where $\lambda=\left\ulcorner\chi_{1}(\sigma), \chi_{2}(\sigma)\right\urcorner$.

The induction step for part (1) of Proposition 3 follows from our proof below that $H \in \mathcal{O}(X)$. The induction step for Clause (2.1) is handled simultaneously with that of part (3).

Induction Step for Clause (2.2) of Proposition 3. Assume $\exists \tau \supseteq \sigma$ such that $\left\langle c p\left(\chi_{1}, \tau\right), c p\left(\chi_{2}, \tau\right)\right\rangle$ is redundant. By Clause (2) of Proposition 2, each of $c p\left(\chi_{1}, \tau\right)$ and $c p\left(\chi_{2}, \tau\right)$ belongs to $\mathcal{O}(\omega)$, and since $\mathcal{O}(\omega)$ excludes constant functions and the identity, we have that $F, G \in \mathcal{O}(X)$. We also have by Corollary 2 of Proposition 2 and the recursive construction of block functions noted above, that the $\theta$ evaluation of the minimal argument that returns $F$ (respectively, $G$ ) projects to the first (respectively, second) coordinate in the last step. By Clause (2.1) of Proposition 3 for the Induction Hypothesis, $F$ has a unique companion $F_{0}$. Let $\chi_{0}$ be a block function such that $\tilde{\chi}_{0}=F_{0}$. The minimal argument for $F_{0}$ is the same as the minimal argument for $F$ except for the direction of the projection. Therefore, $\operatorname{dom}\left(F_{0}\right)=\mathcal{B}(\sigma)$, and $c p\left(\chi_{0}, \tau\right)$ and $c p\left(\chi_{1}, \tau\right)$ are companions. By Corollary 1 of Proposition 2, $c p\left(\chi_{0}, \tau\right)=c p\left(\chi_{2}, \tau\right)$. By part (3) of Proposition 3 for the Induction Hypothesis, $F_{0}=G$; this contradicts that $\langle F, G\rangle$ is not redundant. 
Induction Step for Part (1) and Clause (2.3) of Proposition 3. We have three cases: (i) $F \in g s(X)$ such that $F$ is constant with range $\{f\} \subseteq X$; (ii) $F \in g s(X)$ such that $F$ is the identity on $\mathcal{B}(\sigma)$; and, (iii) $F \in s c(X) \backslash g s(X)$. Then for all $\tau \supseteq \sigma, c p\left(\chi_{1}, \tau\right)$ is: (i) constant with range $\{f(h e i g h t(\tau))\}$; (ii) the identity on $\omega$; and, in the last case, (iii) a member of $\mathcal{O}(M)$ where $M=\{h(h e i g h t(\tau)): h \in X\}$ (by Clause (2.3) of Proposition 3 for the Induction Hypothesis). The same of course is true of $G$. Therefore, for all $\tau \supseteq \sigma$, at least one of $c p\left(\chi_{1}, \tau\right)$ and $c p\left(\chi_{2}, \tau\right)$ is nonconstant (since at least one of $F$ and $G$ is nonconstant), and each belongs to $s c(M)$. Thus, we can conclude that $c p(\chi, \tau) \in \mathcal{O}(M)$ for the following reasons: (i) the Induction Step for Clause (2.2); (ii) $c p(\psi, \gamma) \neq I d$ where $\gamma=\left\ulcorner\chi_{1}(\tau), \chi_{2}(\tau)\right\urcorner$ (see Fact 2); and, (iii) Corollary 1 to Proposition 2.

The preceding result implies that $H \in \mathcal{O}(X)$, since one of (in fact, all of) its component functions belongs to $\mathcal{O}(\omega)$.

Induction Step for Clause (2.4) of Proposition 3. Suppose $\tau_{1}, \tau_{2} \in \Sigma$ are distinct extensions of $\sigma$ with height $\left(\tau_{1}\right)=\operatorname{height}\left(\tau_{2}\right)=m$. Let $\gamma_{1}=\left\ulcorner\chi_{1}\left(\tau_{1}\right), \chi_{2}\left(\tau_{1}\right)\right\urcorner$ and let $\gamma_{2}=\left\ulcorner\chi_{1}\left(\tau_{2}\right), \chi_{2}\left(\tau_{2}\right)\right\urcorner$. Suppose $F, G \in g s(X)$. Then at least one of $\chi_{1}$ and $\chi_{2}$ is the identity on $\operatorname{ext}(\sigma)$, and so $\gamma_{1} \neq \gamma_{2}$. By Clause (4) of Fact 1, $\operatorname{subgroup}\left(\Delta, \gamma_{1}\right)$ and $\operatorname{subgroup}\left(\Delta, \gamma_{2}\right)$ have only the identity in common; therefore, $\left\{\eta(t): t \in \omega^{2}\right.$ is a root \& $\left.\eta \in \operatorname{subgroup}\left(\Delta, \gamma_{1}\right) \& \eta \neq I d\right\}$ and $\{\eta(t): t \in$ $\omega^{2}$ is a root \& $\left.\eta \in \operatorname{subgroup}\left(\Delta, \gamma_{2}\right) \& \eta \neq I d\right\}$ are disjoint subsets of $R$. This fact combined with $\operatorname{cp}\left(\psi, \gamma_{1}\right) \in \operatorname{subgroup}\left(\Delta, \gamma_{1}\right) \backslash\{I d\}, \operatorname{cp}\left(\psi, \gamma_{2}\right) \in \operatorname{subgroup}\left(\Delta, \gamma_{2}\right) \backslash$ $\{I d\}$, the Induction Step for Clause (2.2) of Proposition 3 (which guarantees that redundancy does not occur), and Clause (6) of Proposition 2, implies that $\left\{c p\left(\chi, \tau_{1}\right)\right.$ $(n): n \in \omega \backslash L\}$ and $\left\{c p\left(\chi, \tau_{2}\right)(n): n \in \omega \backslash L\right\}$ are disjoint, where $L=C l_{\Delta}(\{h(m)$ : $h \in X\})$. Suppose that at least one of $F$ and $G$ is not in $g s(X)$. Then $\left\{\left\langle m_{1}, m_{2}\right\rangle\right.$ : $\left.\exists n \in \omega \backslash L\left[m_{1}=c p\left(\chi_{1}, \tau_{1}\right)(n) \& m_{2}=c p\left(\chi_{2}, \tau_{1}\right)(n)\right]\right\}$ and $\left\{\left\langle m_{1}, m_{2}\right\rangle: \exists n \in\right.$ $\left.\omega \backslash L\left[m_{1}=c p\left(\chi_{1}, \tau_{2}\right)(n) \& m_{2}=c p\left(\chi_{2}, \tau_{2}\right)(n)\right]\right\}$ are disjoint subsets of $\omega^{2} \backslash R$. Disjointness follows from Clause (2.4) of Proposition 3 for the Induction Hypothesis. Inclusion in $\omega^{2} \backslash R$ follows from the Induction Step for Clause (2.2) of Proposition 3 (which guarantees that redundancy does not occur), the Induction Hypothesis for Clause (2.3) of Proposition 3 (which guarantees at least one nonconstant component in the construction of each set), and Clause (6) of Proposition 2.

Induction Step for Clause (2.1) and Part (3) of Proposition 3. Suppose $H^{\prime} \in \operatorname{sc}(X)$ with $\operatorname{lev}\left(H^{\prime}\right) \leq k+1$, and let $\chi^{\prime}$ be a block function with $\tilde{\chi}^{\prime}=H^{\prime}$. Suppose $\operatorname{dom}\left(H^{\prime}\right)=\mathcal{B}(\sigma)$. If $H^{\prime} \in g s(X)$, then $\forall \tau \in \Sigma$ with $\tau \supseteq \sigma, \operatorname{cp}\left(\chi^{\prime}, \tau\right) \in$ $g s(\omega)$; whereas, $c p(\chi, \tau) \in \mathcal{O}(\omega)$ by the Induction Step for Clause (2.3).

Suppose $H^{\prime} \notin g s(X)$. Let $\left\langle i^{\prime}, \psi^{\prime}, F^{\prime}, G^{\prime}\right\rangle$ be a minimal $\theta$ argument for $H^{\prime}$. We have, therefore, that $\theta\left(i^{\prime}, \psi^{\prime}, F^{\prime}, G^{\prime}\right)=H^{\prime}$, and the level of $H^{\prime}$ exceeds that of both $F^{\prime}$ and $G^{\prime}$. Then the following are equivalent:

(i) $\exists \tau \in \Sigma$ with $\tau \supseteq \sigma$ such that $c p\left(\chi^{\prime}, \tau\right)=c p(\chi, \tau)$;

(ii) $\left\langle i^{\prime}, \psi^{\prime} \mid \operatorname{ext}(\lambda), F^{\prime}, G^{\prime}\right\rangle=\langle i, \psi \mid \operatorname{ext}(\lambda), F, G\rangle$;

(iii) $H^{\prime}=H$.

(i) implies (ii). Let $\chi_{1}^{\prime}$ and $\chi_{2}^{\prime}$ be block functions with $\tilde{\chi}_{1}^{\prime}=F^{\prime}$ and $\tilde{\chi}_{2}^{\prime}=$ $G^{\prime}$. Let $\gamma=\left\ulcorner\chi_{1}(\tau), \chi_{2}(\tau)\right\urcorner$, and let $\gamma^{\prime}=\left\ulcorner\chi_{1}^{\prime}(\tau), \chi_{2}^{\prime}(\tau)\right\urcorner$. Since we are working with minimal $\theta$ arguments, $c p(\psi, \gamma) \neq I d$ and $c p\left(\psi^{\prime}, \gamma^{\prime}\right) \neq I d$. By the recursive construction of block functions noted above, the fact that $\theta_{0}$ is one-to-one (Corollary 1 to Proposition 2), and the Induction Step for Clause (2.2) of Proposition 3, 
the hypothesis implies that $i^{\prime}=i, c p\left(\psi^{\prime}, \gamma^{\prime}\right)=c p(\psi, \gamma), c p\left(\chi_{1}^{\prime}, \tau\right)=c p\left(\chi_{1}, \tau\right)$, and $c p\left(\chi_{2}^{\prime}, \tau\right)=c p\left(\chi_{2}, \tau\right)$. By Clause (4) of Fact 1 and Clause (1) of Fact 2, $\gamma^{\prime}=\gamma$; and, by Clause (2) of Fact 2, $\psi^{\prime}|\operatorname{ext}(\lambda)=\psi| \operatorname{ext}(\lambda)$. If $\operatorname{cp}\left(\chi_{1}, \tau\right)$ is constant, then $F$ is constant with range $\{f\} \subseteq X$, and $F^{\prime}$ is constant with range $\left\{f^{\prime}\right\} \subseteq X$. Since $f \mid[0$, height $(\tau)]=f^{\prime} \mid[0$, height $(\tau)]$ (by $\left.\gamma=\gamma^{\prime}\right)$ and height $(\tau) \geq \operatorname{split}(X), f^{\prime}=f$; so $F^{\prime}=F$. If $c p\left(\chi_{1}, \tau\right)$ is the identity on $\omega$, then $F^{\prime}=F=$ Identity on $\mathcal{B}(\sigma)$. The same argument applies to $G$ and $G^{\prime}$. Suppose that each of the four component functions belongs to $\mathcal{O}(\omega)$. Then $\left\{F, F^{\prime}, G, G^{\prime}\right\} \subseteq s c(X) \backslash g s(X)$. By Part (3) of Proposition 3 for the Induction Hypthesis, $F^{\prime}=F$ and $G^{\prime}=G$.

(ii) implies (iii). $\theta$ is a well-defined single-valued function.

(iii) implies (i). Tilde is one-to-one giving us $\chi^{\prime}=\chi$; in turn, this implies that $c p\left(\chi^{\prime}, \tau\right)=c p(\chi, \tau)$ for all $\tau \supseteq \sigma$. (ii).

Part (3) follows from (i) implies (iii), and Clause (2.1) follows from (iii) implies

\section{Construction}

8.1. Characterizing the group closure of subsets of the irrationals. Recall that we will recursively construct $X_{\mathbf{c}}$ over $\mathbf{c}$ many stages where at each stage $\alpha \in \mathbf{c}$, one particular potential homeomorphism is killed off by a witness $\left\langle p_{\alpha}, q_{\alpha}\right\rangle$; the homeomorphism maps $p_{\alpha}$ to $q_{\alpha}, p_{\alpha}$ is adjoined, and $q_{\alpha}$ is permanently excluded. The problem is to insure that after adjoining $p_{\alpha}$ and then taking the $\mathcal{G}$ closure, we obtain a set disjoint from $\left\{q_{\beta}: \beta \leq \alpha\right\}$. Lemma 3 serves this purpose by characterizing the $\mathcal{G}$ closure in terms of evaluations of special operators at the single argument $p_{\alpha}$.

Fact 5. Suppose $X \subseteq \mathcal{P}_{1}$ with $\mathcal{D} \subseteq X$. Then $|\mathcal{O}(X)|=|s c(X)|=|g s(X)|=|X|$.

Lemma 3. Suppose $X \subseteq \mathcal{P}_{1}$ with $\mathcal{D} \subseteq X$, and $f \in \mathcal{P}_{1}$. Then $C l_{\mathcal{G}}(X \cup\{f\}) \subseteq$ $C l_{\mathcal{G}}(X) \cup\{f\} \cup\left\{F(f): F \in \mathcal{O}\left(C l_{\mathcal{G}}(X)\right) \& f \in \operatorname{dom}(F)\right\}$.

Proof of Lemma 3. Let $Y=C l_{\mathcal{G}}(X) \cup\{f\} \cup\left\{F(f): F \in \mathcal{O}\left(C l_{\mathcal{G}}(X)\right)\right.$ \& $f \in$ $\operatorname{dom}(F)\}$. Let $g, h \in Y, \psi \in \mathcal{G}$, and $i \in\{1,2\}$. We need to show that $\tilde{\psi}(\ulcorner Y \times Y\urcorner) \subseteq$ $\ulcorner Y \times Y\urcorner$, which is equivalent to showing that $\pi_{i} \circ(\tilde{\psi}(\ulcorner g, h\urcorner)) \in Y$. Choose $G \in$ $\operatorname{sc}\left(X_{1}\right)$ and $H \in \operatorname{sc}\left(X_{2}\right)$, where $X_{1}$ and $X_{2}$ are finite subsets of $C l_{\mathcal{G}}(X)$ such that $f \in$ $\mathcal{B}(\sigma) \cap \mathcal{B}(\tau)$ where $\sigma, \tau \in \Sigma$ with $\mathcal{B}(\sigma)=\operatorname{dom}(G)$ and $\mathcal{B}(\tau)=\operatorname{dom}(H), g=G(f)$, and $h=H(f)$. If $g$ belongs to $C l_{\mathcal{G}}(X)$, then $G$ is constant, and if $g=f$, then $G$ is the identity (of course, the same holds for $h$ and $H$ ). Since $\sigma$ and $\tau$ are comparable, we can choose $\rho \supseteq \sigma \cup \tau$ in $\Sigma$ such that height $(\rho) \geq \max \left\{\operatorname{split}\left(X_{1} \cup X_{2}\right)\right.$, length $\left.(\psi)\right\}$ and $f \in \mathcal{B}(\rho)$. Now fix $F$ by the definition $F=\theta(i, \psi, G|\mathcal{B}(\rho), H| \mathcal{B}(\rho))$. Then $F(f)=\pi_{i} \circ(\tilde{\psi}(\ulcorner g, h\urcorner))$. If each of $G \mid \mathcal{B}(\rho)$ and $H \mid \mathcal{B}(\rho)$ is constant, then $F(f) \in C l_{\mathcal{G}}(X)$; otherwise, $F \in s c\left(C l_{\mathcal{G}}(X)\right)$. So by Part (1) of Proposition 3, $F \in \mathcal{O}\left(C l_{\mathcal{G}}(X)\right)$ or $F(f) \in C l_{\mathcal{G}}(X) \cup\{f\}$.

8.2. Special operators and bounded cartesian products. The last step is a constraint on the ranges and point-inverse sets of special operators that (combined with Lemmas 1 and 3) guarantees that our recursive construction can be continued through c many stages.

Lemma 4. Suppose $X \subseteq \mathcal{P}_{1}$ is finite, $F \in \mathcal{O}(X)$, and $Y \subseteq \mathcal{P}_{1}$ is either the range of $F$ or some point-inverse set of $F$. Then $Y$ is a subset of a bounded cartesian product. 
Proof of Lemma 4. Let $\sigma \in \Sigma$ and let $\chi$ be a block function such that $\operatorname{dom}(F)=$ $\mathcal{B}(\sigma)$ and $\tilde{\chi}=F$. Let $\tau=\chi(\sigma)$. Note that height $(\tau)=$ height $(\sigma)$. For all $n<$ height $(\sigma)$, let factor $(n)=\{\tau(n)\}$, and, for all $n \geq$ height $(\sigma)$, let factor $(n)=$ $\exp _{2}(\{h(n): h \in X\}) \cup E_{1} \cup E_{2}$. Let $m=2|X|$. By Clause (1) of Proposition 1 , for all $n \in \omega\left[\mid\right.$ factor $\left.(n) \cap E_{0} \mid \leq m\right]$. By the combined effect of Clause (2) of Proposition 1, Clauses (1) and (3) of Proposition 2, and Clause (2.3) of Proposition $3, \operatorname{ran}(F) \subseteq \Pi_{n \in \omega}$ factor $(n)$.

Let $f \in \operatorname{ran}(F)$. We need two alterations in the definition of factor to obtain a product that includes $F^{-1}(f)$ as a subset. For all $n<$ height $(\sigma)$, let $f a c t o r '(n)=$ $\{\sigma(n)\}$. Suppose $n \geq$ height $(\sigma)$. Let $M=\{h(n): h \in X\}$, and define factor $^{\prime}(n)=$ $\exp _{2}(M) \cup E_{1} \cup E_{2} \cup\{k \in \omega \backslash L: \exists \rho \in \Sigma$ with $\rho \supseteq \sigma[$ height $(\rho)=n \& \operatorname{cp}(\chi, \rho)(k)=$ $f(n)]\}$ where $L=C l_{\Delta}(M)$; with respect to the last term of this union, there is at most one $\rho$ by Clause (2.4) of Proposition 3, and given $\rho$, there is at most one $k$ by Clause (4) of Proposition 2 (so we obtain factor $^{\prime}(n)$ by adjoining at most one point to factor $(n))$. We now have that $\forall n \in \omega\left[\mid\right.$ factor $\left.^{\prime}(n) \cap E_{0} \mid \leq m+1\right]$, and $F^{-1}(f) \subseteq \Pi_{n \in \omega}$ factor $^{\prime}(n)$.

8.3. Proof of the Theorem. We now construct our rigid space with homogeneous square as $X_{\mathbf{c}}=C l_{\mathcal{G}}\left(\mathcal{D} \cup\left\{p_{\alpha}: \alpha \in \mathbf{c}\right\}\right)$ where $\left\langle p_{\alpha}: \alpha \in \mathbf{c}\right\rangle$ has a recursive definition. Let $\left\langle H_{\alpha}: \alpha \in \mathbf{c}\right\rangle$ be a well-ordering of $\mathcal{H} \backslash\{$ Identity Function on $\mathcal{D}\}$. At each stage $\alpha$, we choose $p_{\alpha} \in \operatorname{dom}\left(H_{\alpha}^{e}\right)$ so that if $q_{\alpha}=H_{\alpha}^{e}\left(p_{\alpha}\right)$, then $\left\{q_{\beta}: \beta \leq \alpha\right\}$ is disjoint from the $\mathcal{G}$ closure of $\mathcal{D} \cup\left\{p_{\beta}: \beta \leq \alpha\right\}$.

Note that at the outset we commit each point of $\mathcal{D}$ to membership in our objective space, and that $\forall \alpha \in \mathbf{c}$, we set $q_{\alpha}=H_{\alpha}^{e}\left(p_{\alpha}\right)$ by definition.

Let $\alpha \in \mathbf{c}$, and let $X_{\alpha}=C l_{\mathcal{G}}\left(\mathcal{D} \cup\left\{p_{\beta}: \beta<\alpha\right\}\right)$. By Fact $3,\left|X_{\alpha}\right|<\mathbf{c}$. For the Recursion Hypothesis, suppose that $X_{\alpha}$ is disjoint from $\left\{q_{\beta}: \beta<\alpha\right\}$.

Let $\nu \in \Sigma$ such that $\mathcal{D} \cap \mathcal{B}(\nu)$ is disjoint from its image under $H_{\alpha}$. Let $j$ be a strictly increasing sequence with $\operatorname{ran}(j)=E_{0}$. In reference to $H_{\alpha}, \mathcal{B}(\nu)$, and $j$, choose $T \subseteq \operatorname{dom}\left(H_{\alpha}^{e}\right) \cap \mathcal{B}(\nu)$ according to Lemma 1 . Then $|T|=\mathbf{c}$, whereas each of $T$ and $H_{\alpha}^{e}[T]$ has finite intersection with every bounded cartesian product. By Lemma 4, each member of $\mathcal{C}=\left\{\operatorname{ran}(F): F \in \mathcal{O}\left(X_{\alpha}\right)\right\} \cup\left\{F^{-1}\left(q_{\beta}\right): \beta<\alpha \& F \in\right.$ $\left.\mathcal{O}\left(X_{\alpha}\right)\right\}$ is a subset of a bounded cartesian product; and since $\left|\mathcal{O}\left(X_{\alpha}\right)\right|=\left|X_{\alpha}\right|$ by Fact 5 , we have that $|\mathcal{C}|<\mathbf{c}$. We can therefore choose $p_{\alpha} \in T$ so that each of $p_{\alpha}$ and $q_{\alpha}=H_{\alpha}^{e}\left(p_{\alpha}\right)$ belongs to the complement of $\left((\cup \mathcal{C}) \cup X_{\alpha} \cup\left\{q_{\beta}: \beta<\alpha\right\}\right)$. By Lemma 3, the Recursion Hypothesis is preserved. Let $X_{\mathbf{c}}=C l_{\mathcal{G}}\left(\mathcal{D} \cup\left\{p_{\alpha}: \alpha \in \mathbf{c}\right\}\right)$.

Note that $X_{\mathbf{c}}=\bigcup_{\alpha \in \mathbf{c}} X_{\alpha}$; therefore, $X_{\mathbf{c}}$ is rigid by construction. The square, $X_{\mathbf{c}} \times X_{\mathbf{c}}$, is homogeneous by Lemma 2 .

\section{Problems}

Problem 1. Is it possible to construct a dense rigid subspace of the real line without using transfinite recursion? In particular, is there a rigid Borel subset of the real line?

Problem 2. Replace rigid by van Mill (i.e., rigid with homogeneous square) in Problem 1.

Problem 3. The example of this paper has cardinality c. Is there a consistency example of a dense rigid subspace of the real line with cardinality less than $\mathbf{c}$ ? Recall that the Proper Forcing Axiom implies that any two $\aleph_{1}$ dense (i.e., cardinality $\aleph_{1}$ in each open interval) subsets of the real line are order-isomorphic. 
Problem 4 (J. Roitman). Is there a consistency example of a van Mill space with cardinality less than $\mathbf{c}$ ?

Problem 5 (R. Levy, J. van Mill and M. E. Rudin). We first need a definition: As usual, a power of a topological space $X$ is a cartesian product (with the Tychonoff product topology of pointwise convergence) where every factor space is $X$; a power (with finitely many factors) of a topological space is almost rigid iff the only homeomorphisms of the power onto itself are those that are induced by a permutation of the index set. Is there, for each positive integer $n$, a subspace $X$ of the real line such that for each positive $m \leq n, X^{m}$ is almost rigid, whereas $X^{n+1}$ is homogeneous? For $n=1$, this is, of course, van Mill's Problem.

\section{ACKNOWLEDGEMENT}

The author thanks the referee for the careful review of this paper.

\section{REFERENCES}

1. Jan van Mill, $A$ rigid space $X$ for which $X \times X$ is homogeneous; an application of infinitedimensional topology, Proc. Amer. Math. Soc. 83 (1981), 597-600. Mr $82 \mathrm{~h}: 54067$

Department of Mathematics, George Mason University, Fairfax, Virginia 22030-4444

E-mail address: blawrenc@mail.gmu .edu 